SubmitTed to ApJ

Preprint typeset using LTEX style emulateapj v. 2/16/10

\title{
ON THE CLUSTER PHYSICS OF SUNYAEV-ZEL'DOVICH AND X-RAY SURVEYS III: MEASUREMENT BIASES AND COSMOLOGICAL EVOLUTION OF GAS AND STELLAR MASS FRACTIONS
}

\author{
N. Battaglia ${ }^{1}$, J. R. Bond ${ }^{2}$, C. Pfrommer ${ }^{3, *}$, J. L. Sievers ${ }^{4,5}$ \\ ${ }^{1}$ McWilliams Center for Cosmology, Carnegie Mellon University, Department of Physics, 5000 Forbes Ave., Pittsburgh PA, USA, 15213 \\ ${ }^{2}$ Canadian Institute for Theoretical Astrophysics, 60 St George, Toronto ON, Canada, M5S 3H8 \\ ${ }^{3}$ Heidelberg Institute for Theoretical Studies, Schloss-Wolfsbrunnenweg 35, D-69118 Heidelberg, Germany \\ ${ }^{4}$ Joseph Henry Laboratories of Physics, Jadwin Hall, Princeton University, Princeton NJ, USA, 08544 \\ ${ }^{5}$ Astrophysics and Cosmology Research Unit, University of Kwazulu-Natal, Westville, Durban 4000, South Africa \\ corresponding author, christoph.pfrommer@h-its.org \\ Submitted to ApJ
}

\begin{abstract}
Gas masses tightly correlate with the virial masses of galaxy clusters, allowing for a precise determination of cosmological parameters by means of large-scale X-ray surveys. However, the gas mass fractions $\left(f_{\text {gas }}\right)$ at the virial radius $\left(R_{200}\right)$ derived from recent Suzaku observations of several groups are considerably larger than the cosmic mean, calling into question the accuracy of estimates of cosmological parameters. Here, we use a large suite of cosmological hydrodynamical simulations to study measurement biases of $f_{\text {gas }}$. We employ different variants of simulated physics, including radiative gas physics, star formation, and thermal feedback by active galactic nuclei, which we show is able to arrest overcooling and to result in constant stellar mass fractions for redshifts $z<1$. This implies that the stellar and dark matter masses increase at the same rate, which is realized if most of the stellar mass is already in place by the time it assembles in the cluster halo-in agreement with observations. Computing the mass profiles in 48 angular cones, whose footprints partition the sphere, we find anisotropic gas and total mass distributions that imply an angular variance of $f_{\text {gas }}$ at the level of $30 \%$. This anisotropic distribution originates from the recent formation epoch of clusters and from the strong internal baryon-to-dark-matter density bias. In the most extreme cones, $f_{\text {gas }}$ can be biased high by a factor of two at $R_{200}$ in massive clusters $\left(M_{200} \sim 10^{15} \mathrm{M}_{\odot}\right)$, thereby providing a potential explanation for high $f_{\text {gas }}$ measurements by Suzaku. While projection lowers this factor, there are other measurement biases that may (partially) compensate. We find that at $R_{200}, f_{\text {gas }}$ is biased high by $20 \%$ when assuming hydrostatic equilibrium masses, i.e., neglecting the kinetic pressure, and by another $\sim 10-20 \%$ due to the presence of density clumping (depending on mass and dynamical state). At larger radii, both measurement biases increase dramatically. While the cluster sample variance of the true $f_{\text {gas }}$ decreases to a level of $5 \%$ at $R_{200}$, the sample variance that includes both measurement biases remains fairly constant at the level of $10-20 \%$ (depending on dynamical state). At the high-mass end $\left(M_{500}>2 \times 10^{14} \mathrm{M}_{\odot}\right)$, the true $f_{\text {gas }}$ within $R_{500}$ shows a constant redshift evolution. While this result is in principle encouraging for using gas masses to derive cosmological parameters, careful X-ray mocks are needed to control those various measurement biases.

Subject headings: Cosmology: Theory — Galaxies: Clusters: General — Large-Scale Structure of Universe — Methods: Numerical
\end{abstract}

\section{INTRODUCTION}

Galaxy clusters are the rarest and largest gravitationallycollapsed objects in the universe, making them very sensitive tracers of the growth of structure (for a review, see Voit 2005). Clusters exhibit a well-defined number count that steeply falls as mass and redshift increase. The number density tail is very sensitive to changes in cosmological parameters (as demonstrated by recent measurements, e.g., by Allen et al. 2008; Vikhlinin et al. 2009b) including the dynamical characteristics of dark energy, primordial non-Gaussianity, and the theory of gravity. Hence, future large surveys of clusters (in the optical, X-ray, and microwave/radio/sub-mm wavelengths) with substantially increased statistics and resolution can potentially provide a gold mine for fundamental cosmology (e.g., Battye \& Weller|2003; Hu 2003; Maiumdar \& Mohr 2003, 2004; Khedekar et al. 2010; Galli et al. 2012). However, this is only possible if systematics associated with cluster mass calibrations and our incomplete knowledge of the physics of the intracluster medium (ICM) can be understood and controlled. As observations have been progressively refined and resolutions improved, the clusters have been revealed to be too complex for simple sphericalized analytical modelling, calling for detailed theoretical work with a necessarily heavy computational component.

The virialized regions of clusters - usually characterized by a sphere of radius $R_{200}$ enclosing a mean density that is 200 times the critical density of the Universe (and higher mean interior densities correspond to smaller radii) - are separated from the Hubble-flow. However, those regions maintain contact with the surrounding filamentary cosmic web through ongoing accretion and mergers as they evolve. Most of the cluster baryons are in the form of a hot $(k T \sim(1-10) \mathrm{keV})$ diffuse plasma, the ICM, while the remaining baryons are confined to the cluster's numerous stars and galaxies. Since cold dark matter (CDM) obeys the collisionless Boltzmann equation and since the dynamics of the hot baryons additionally responds to pressure forces according to the (collisional) Euler equation (neglecting viscosity effects), we expect a strong internal baryon-to-darkmatter (DM) density bias. We hope that when averaged over cluster scales, the smoothed densities are nearly in the universal Hubble-volume-smoothed proportion (or a constant, large fraction thereof). But at which radius and cluster scale this exactly happens depends on baryonic physics and needs to be 
carefully studied using a combination of hydrodynamical cosmological simulations and ICM observations.

Using Chandra X-ray observations of a sample of relaxed clusters, earlier work has successfully used the gas mass fraction in clusters to derive cosmological parameters in agreement with the concordance $\Lambda$ CDM model Allen et al. 2002, 2004, 2008). In these analyses, masses were derived assuming spherical symmetry and hydrostatic equilibrium while allowing for a modest constant non-thermal pressure contribution. Similar conclusions were obtained through a joint X-ray and Sunyaev-Zel'dovich (SZ) analysis (LaRoque et al. 2006) as well as in a Chandra sample of X-ray luminous clusters extending to a redshift of $z \lesssim 1.3$ (Ettori et al. 2009). Recently, a more sophisticated analysis of an X-ray flux-selected sample of 238 clusters at $z \leq 0.5$, which takes into account various selection effects, combines gas mass fractions, X-ray luminosity, and temperature (Mantz et al. 2010c a). These authors derive improved cosmological parameters that are consistent with $\Lambda C D M$ and have approximately equal constraining power compared to other cosmological probes (at the time of publication) on the DM density parameter, $\Omega_{m}$, the rms amplitude of the (linear) density power spectrum on cluster-mass scales, $\sigma_{8}$, and the equation of state of dark energy. These data were even used to obtain interesting constraints on modified gravity and general relativity on cosmological scales (Rapetti et al. 2009, 2010) as well as on neutrino properties (Mantz et al. 2010b). However, in order to get unbiased cosmological constraints, the gas mass fraction, $f_{\text {gas }}=M_{\text {gas }} / M_{\text {tot }}$, either needs to be independent of both, redshift and mass (Sadat et al. 2005) or, if $f_{\text {gas }}$ varied with redshift and/or mass, the associated biases and the scatter around them would have to be accurately quantified.

Vikhlinin et al. (2006) studied gas and total mass profiles in a small sample of Chandra clusters of high-quality data out to $R_{500}$. They find that enclosed $f_{\text {gas }}$ profiles within $R_{2500} \simeq$ $0.4 R_{500}$ do not asymptotically approach the universal baryon fraction derived from cosmic microwave background (CMB) observations (with a discrepancy of a factor of 1.5-2). This discrepancy becomes smaller with increasing cluster mass and radius, but is still noticeable at $R_{500}$ enforcing the need to understand the behavior of $f_{\text {gas }}$ at even larger radii.

The recently launched Suzaku has enabled measurements of cluster X-ray emission up to and even beyond $R_{200}$ due to its low-Earth orbit that ensures a low and stable particle background, almost an order of magnitude lower than previous $\mathrm{X}$ ray telescopes. These measurements are critical in improving our understanding of the formation history of galaxy clusters, the dynamical state of the ICM at greater radii without the need to extrapolate measurements of the core regions of clusters, and to gain confidence in assumptions used to derive cosmological parameters from cluster surveys. There is now a growing collection of Suzaku clusters, consisting of PKS0745191 (George et al. 2009), Abell 1795 (Bautz et al. 2009), Abell 2204 (Reiprich et al. 2009), Abell 1413 (Hoshino et al. 2010), Abell 1689 (Kawaharada et al. 2010), Abell 2142 (Akamatsu et al. 2011), Perseus (Simionescu et al. 2011), a fossil group RX J1159+5531 (Humphrey et al. 2012), Abell 2029 (Walker et al. 2012), and Hydra A (Sato et al. 2012).

While most of these Suzaku measurements observe an excess $\mathrm{X}$-ray emission for radii $r \gtrsim R_{500}$ over what is expected from cosmological hydrodynamical simulations, there are two clusters, Abell 1795 and RX J1159+5531, where the emission is compatible with the theoretical expectations (within the error bars). The excess emission manifests itself in entropy profiles which rise less steeply than the predictions of purely gravita- tional hierarchical structure formation (and can even start to drop beyond $R_{200}$ ). Alternatively, this implies increasing $f_{\text {gas }}$ profiles reaching values above the cosmic mean and, in one case, even boosting the universal baryon fraction by a factor of 1.6 at $R_{200}$ when accounting for a stellar mass fraction of $12 \%$ (Simionescu et al. 2011). In some cases, the excess emission could be explained by the anisotropic nature of the cosmic filaments connecting to clusters, in particular if there is an overlap of the outskirts of two neighboring clusters (as it is possibly the case in Abell 2029). Possible physical explanations include substantial density clumping and/or neglecting (respectively underestimating) non-thermal pressure in deriving hydrostatic masses. Since the X-ray emissivity is proportional to the square of the gas density, a clumpy ICM with dense regions will induce X-ray surface brightness fluctuations (Churazov et al. 2012; Sanders \& Fabian 2012) that enhance the emission over the smooth component, biasing the entropy lower and $f_{\text {gas }}$ higher in comparison to an extrapolation from the more homogeneous inner regions. Observational or instrumental reasons for the observed excess emission (which is typically a factor of 3-5 below the soft extragalactic X-ray background) include fluctuations of unresolved point sources, point-spread function leakage from masked point sources, or stray light from the bright inner cluster core.

To better address possible systematics, a large sample of clusters with well-defined selection criteria are currently being observed, exploiting the strengths of three complementary X-ray observatories: Suzaku (low, stable background), $X M M-N e w t o n$ (high effective area and sensitivity), and Chandra (good spatial resolution) (Miller et al. 2012). Combining Chandra and Suzaku pointings in cluster outskirts increases the number of detected point sources by a factor of 10 and, hence, reduces the uncertainty on the X-ray surface brightness by a factor of $\sim 3$. Early results from this program on Abell 3378 show entropy and $f_{\text {gas }}$ profiles that are in fact compatible with theoretical expectations. This result is confirmed by a stacking analysis of ROSAT clusters that detects a steepening of the density profiles beyond $\sim R_{500}$ (Eckert et al. 2012), in agreement with simulation results that include radiative physics and account for gas clumping (Nagai \& Lau 2011) - apparently contradicting recent $S u z a k u$ results.

Hydrodynamical simulations of the formation of clusters that include radiative cooling, star formation and stellar feedback predict stellar mass fractions in excess of what is observed, a decreasing gas mass fraction with redshift, and overpredict the X-ray luminosity on group scales (Kravtsov et al. 2005; Ettori et al.2006; Roncarelli et al. 2006). This problem is substantially mitigated when including energetic feedback by active galactic nuclei (AGN) (Siiacki \& Springel 2006; Sijacki et al. 2007, 2008; Puchwein et al. 2008; Booth \& Schave 2009; Fabjan et al. 2010; McCarthy et al. 2010; Dubois et al. 2010; Teyssier et al. 2011; Young et al. 2011; Planelles et al. 2013). Interestingly, including AGN feedback brings the modeled SZ power spectrum into agreement with data while purely radiative models overproduce the measured power on scales around 3 arcmin $(\ell \sim 3000)$ (Battaglia et al. 2010, 2012b; Shaw et al. 2010; Trac et al. 2011). Most notably, the SZ power spectrum of models that only account for shock heating and neglect radiative physics and hence the condensation of baryons into stars produce too much power on scales of $\ell \sim 3000$ (Battaglia et al. 2010), in strong tension with the measurements by SPT and ACT (Reichardt et al. 2012; Sievers et al. 2013). While the gas mass fractions in these models are higher than in the corre- 
sponding radiative models (with and without AGN feedback), they are somewhat lower than the universal baryon fractions. This demonstrates that for consistency reasons with the SZ power spectrum, the $S u z a k u$-inferred high values of $f_{\text {gas }}$ along some radial arms either are not representative for the entire virial regions of those clusters or appear to be biased high. Hence this emphasizes that a successful model of the cluster physics not only needs to match stellar and gas mass fractions as a function of radius and cluster mass, but also has to adhere to constraints from SZ surveys, namely scaling relations and power spectrum measurements.

This is the third in a series of papers addressing the cluster physics of SZ and X-ray surveys. In the first two papers, we thoroughly scrutinized the influence of feedback, nonthermal pressure and cluster shapes on $Y-M$ scaling relations (Battaglia et al. 2012a, hereafter BBPS1) and the thermal SZ power spectrum (Battaglia et al. 2012b, hereafter BBPS2). The fourth paper will detail the physics of density and pressure clumping due to infalling substructures, accompanying the growth of clusters (Battaglia et al. 2013a, hereafter BBPS4), and the fifth will provide an information theoretic view of clusters and their non-equilibrium entropies (Battaglia et al.2013b, hereafter BBPS5).

In this work, we are motivated by current and next generation surveys in the X-rays (eROSITA) and those employing the Sunyaev-Zel'dovich effect such as the Atacama Cosmology Telescope (ACT, Fowler et al. 2007), the South Pole Telescope (SPT, Carlstrom et al. 2011), and Planck to reassess critically the impact of structure formation and physics on $f_{\text {gas }}$ measurements, in particular in the outskirts of galaxy clusters. Using a large suite of cosmological simulations, we first quantify the bias of $f_{\text {gas }}$ measurements due to non-equilibrium processes in the cluster outskirts while allowing for cluster sample variance. Then, we provide an extensive study of $f_{\text {gas }}$ in our various physical models to obtain a solid theoretical foundation for cluster cosmology using gas mass fractions. In Section 2, we introduce our simulations and modeled physics. In Section 3 . we study how typical assumptions in measuring gas fractions, e.g., adopting hydrostatic mass estimates and neglecting density clumping, bias the measured values. In Section 4 we study how $f_{\text {gas }}$ varies with radius, cluster mass, redshift, and simulated physics, and compare our simulation values to observational data. We conclude in Section 5 .

\section{COSMOLOGICAL SIMULATIONS AND CLUSTER DATA SET}

We use smoothed particle hydrodynamic (SPH) simulations of large-scale periodic boxes which provide us with large cluster samples to accurately characterize ICM properties over large ranges of cluster masses and redshifts. We use a modified version of the GADGET-2 (Springel 2005) code. Our sequence of periodic boxes have lengths of 165 and $330 h^{-1} \mathrm{Mpc}$ filled with $N_{\mathrm{DM}}=N_{\mathrm{gas}}=256^{3}$ and $512^{3}$ dark matter (DM) and gas particles, respectively. This choice maintains the same initial gas particle mass $m_{\text {gas }}=3.2 \times 10^{9} h^{-1} \mathrm{M}_{\odot}$, DM particle mass $m_{\mathrm{DM}}=1.54 \times 10^{10} h^{-1} \mathrm{M}_{\odot}$, and a minimum gravitational smoothing length $\varepsilon_{s}=20 \mathrm{~h}^{-1} \mathrm{kpc}$; our SPH densities were computed with 32 neighbours. For our standard calculations, we adopt a tilted $\Lambda \mathrm{CDM}$ cosmology, with total matter density (in units of the critical) $\Omega_{m}=\Omega_{\mathrm{DM}}+\Omega_{b}=0.25$, baryon density $\Omega_{b}$ $=0.043$, cosmological constant $\Omega_{\Lambda}=0.75$, Hubble parameter $h=0.72$ in units of $100 \mathrm{~km} \mathrm{~s}^{-1} \mathrm{Mpc}^{-1}$, spectral index of the primordial power-spectrum $n_{s}=0.96$ and $\sigma_{8}=0.8$.
We show results from simulations employing three different variants of the the simulated physics: the first only accounts for gravitational shock heating; the second additionally accounts for radiative cooling, star formation, supernova feedback, galactic winds, and cosmic rays physics and is referred to as radiative cooling model; and the last accounts for radiative physics (radiative cooling, star formation, supernova feedback) and thermal feedback by AGNs and is referred to as AGN feedback model. Radiative cooling and heating were computed assuming an optically thin gas of a pure hydrogen and helium primordial composition in a time-dependent, spatially uniform ultraviolet background. Star formation and supernovae feedback were modelled using the hybrid multiphase model for the interstellar medium of Springel \& Hernquist (2003). The CR population is modelled as a relativistic population of protons described by an isotropic power-law distribution function in momentum space with a spectral index of $\alpha=2.3$ (Pfrommer et al. 2006; Enßlin et al. 2007; Jubelgas et al. 2008). With those parameters, the CR pressure modifies the $\mathrm{SZ}$ effect at most at the percent level and causes a reduction of the resulting integrated Compton- $y$ parameter (Pfrommer et al. 2007). The AGN feedback prescription included in the simulations (for more details see Battaglia et al. 2010) allows for lower resolution and hence can be applied to large-scale structure simulations. It couples the black hole accretion rate to the global star formation rate (SFR) of the cluster, as suggested by Thompson et al. (2005). The thermal energy is injected into the ICM such that it is proportional to the star formation within a given central spherical region. Our AGN feedback model produces identical results for $f_{\text {gas }}$ profiles to a more elaborate model (Sijacki et al. 2007, 2008) when run at comparable high resolution (Battaglia et al. 2010) and - by extension - is also able to reconcile the Xray luminosity-temperature scaling relation (Puchwein et al. 2008).

We use a two-step algorithm to compute the virial mass of a cluster. First, we find all clusters in a given snapshot using a friends-of-friends (FOF) algorithm. Then, using a spherical overdensity method with the FOF values as starting estimates, we iteratively calculate the center of mass, the virial radius, $R_{\Delta}$, and mass, $M_{\Delta}$, contained within $R_{\Delta}$, and compute the radially averaged profiles of a given quantity with radii scaled by $R_{\Delta}$. We define $R_{\Delta}$ as the radius at which the mean interior density equals $\Delta$ times the critical density, e.g., for $\Delta=200$ or 500. All cluster profiles are computed as functions of $r / R_{200}$ and are converted to other scaled radii (i.e., in units of $R_{500}$ or $R_{1000}$ ) using the NFW density profile (Navarro et al., 1997), the concentration mass relation from Duffy et al. (2008), and the cosmological parameters listed above. Our cluster sample (for each simulation type) consists of $\simeq 1300$ clusters with $M_{200}>$ $7 \times 10^{13} \mathrm{M}_{\odot}$ and $\simeq 800$ clusters with $M_{200}>1 \times 10^{14} \mathrm{M}_{\odot}$ at $z=0$.

\section{BIASES OF GAS MASS FRACTION MEASUREMENTS}

We are interested in the enclosed gas mass fractions that we define as the ratio of gas mass and total mass inside a given radius, i.e., $f_{\text {gas }}(<r)=M_{\text {gas }}(<r) / M_{\text {tot }}(<r)$. All our values of $f_{\text {gas }}$ and the stellar mass fraction, $f_{\text {star }}$, are normalized by the cosmic baryon fraction $f_{c}=\Omega_{b} / \Omega_{m}$ that should be substituted by the current best fit value from cosmological precision experiments and was assumed to be $f_{c}=0.172$ in our simulations, which is on the high side in comparison to the currently favored value of 0.155 (Planck Collaboration et al. 2013a). Here, we study how typical assumptions in measuring gas fractions, e.g., adopting 
hydrostatic mass estimates and neglecting density clumping, bias the measured values. We additionally address how the sample variance of clusters and the three-dimensional (3D) angular orientation variance within clusters impacts inferred values for $f_{\text {gas }}$. We also address how line-of-side projection effects this angular orientation bias of the gas fraction, i.e., how an $f_{\text {gas }}$ measurement from a mosaic X-ray observation along a radial arm varies with angular orientation depending on whether that arm intersects a (projected) cosmic filament or not.

In Figure1, we show the median enclosed gas mass fractions, $f_{\text {gas }}(<r)$ as a function of radius for different cluster masses in our AGN feedback model; on the left panel for our sample of all clusters, and on the right panel for the 3rd most relaxed clusters. To define a relaxed cluster, we split the population of clusters into lower, middle, and upper bands of the ratio of kinetic-to-thermal energy, $K / U$, within $R_{200}$, which we use as a measure of dynamical state for the clusters (see Appendix A. We define the internal kinetic energy, $K$, and thermal energy, $U$, of a cluster as

$$
\begin{gathered}
K\left(<R_{200}\right) \equiv \sum_{i} \frac{3 m_{\mathrm{gas}, i} P_{\mathrm{kin}, i}}{2 \rho_{i}} \\
U\left(<R_{200}\right) \equiv \sum_{i} \frac{3 m_{\mathrm{gas}, i} P_{\mathrm{th}, i}}{2 \rho_{i}}
\end{gathered}
$$

where $m$ and $\rho$ are the gas mass and the SPH density, respectively for all particles $i$ less than $R_{200}, P_{\text {th }}=k T \rho /\left(\mu m_{p}\right)$ denotes the thermal pressure (where $T$ and $\mu$ denote the temperature and mean molecular weight, respectively), and the kinetic pressure is defined as

$$
\begin{aligned}
P_{\text {kin }} & =\rho \delta \boldsymbol{v}^{2} / 3, \\
\delta \boldsymbol{v} & =a(\boldsymbol{v}-\overline{\boldsymbol{v}})+a H(a)(\boldsymbol{x}-\overline{\boldsymbol{x}}) .
\end{aligned}
$$

The code employs comoving peculiar velocities, which are translated into the internal cluster velocities relative to the overall mean cluster velocity in the Hubble flow by the relation given, where $H(a)$ is the Hubble function, $a$ is the scale factor, $\boldsymbol{v}(=\mathrm{d} \boldsymbol{x} / \mathrm{d} t)$ is the peculiar velocity, and $\boldsymbol{x}$ is the comoving position of each particle. The gas-particle-averaged cluster bulk flow within $R_{200}$ is $\overline{\boldsymbol{v}}$ and the center of mass within $R_{200}$ is $\overline{\boldsymbol{x}}$. Since the most massive clusters are on average also the most disturbed systems, only two out of 25 clusters in our most massive cluster bin $\left(M_{200}>5.1 \times 10^{14} \mathrm{M}_{\odot}\right)$ meet our criterion of being a relaxed cluster, i.e., are among the lowest third when selected by the ratio of kinetic-to-thermal energy, $K / U$. Hence, in order to improve the statistics of our most massive bin of relaxed clusters, we decided to also do a 3 -split on $K / U$ in the largest cluster mass bin.

As shown in Figure 1, $f_{\text {gas }}$ increases with radius and approaches values within $2 R_{200}$ that range from 0.8 to 0.85 (in units of $f_{c}$ ) for the mass interval from $M_{200} \simeq 10^{14} \mathrm{M}_{\odot}$ to $5 \times 10^{14} \mathrm{M}_{\odot}$. Smaller systems show smaller values of $f_{\text {gas }}$ at every radius owing to the shallower potential wells in comparison to bigger systems. In groups and small clusters, AGN feedback is able to push the gas well beyond $R_{200}$ or halt its initial infall. In the process of hierarchical structure formation, these gas-poor groups merge to form larger clusters. Owing to their deeper potentials, those larger clusters provide a greater gravitational pull on the gas that is sitting beyond $R_{200}$. As a result, $f_{\text {gas }}$ increases at each radius in larger clusters. (We will show later on in Section 4.3 that the underlying assumption of a constant $f_{\text {gas }}$ as a function of redshift at fixed cluster mass is justified in our simulations.)
These simulated values of $f_{\text {gas }} / f_{c}<0.8$ for all cluster masses are substantially smaller than Suzaku estimates of $f_{\text {gas }} / f_{c} \sim 1.4$ or even 1.6 within $R_{200}$ if one accounts for a stellar mass fraction of $12 \%$ (Simionescu et al. 2011). Two sources that could potentially bias the measurement of gas fractions have been suggested, the assumption of hydrostatic masses and neglecting density clumping due to infalling (sub-)structures following the ongoing growth of clusters. Hence we will discuss each of these effects in turn.

\subsection{Hydrostatic mass bias}

Even if clusters are in hydrostatic equilibrium (HSE), balancing the gravitational force to the pressure gradient yields

$$
\nabla P=\rho \boldsymbol{g} \rightarrow-\rho G M(<r) \hat{\boldsymbol{r}} / r^{2} \text { for spherical symmetry. }
$$

Assuming that all the pressure in Eq. (5) is thermal $\left(P=P_{\mathrm{th}}\right)$ gives an incorrect estimate for the mass since the non-thermal pressure, in particular the kinetic pressure has to be included (e.g., Evrard 1990; Rasia et al. 2004, 2006; Lau et al. 2009; Rasia et al. 2012; Nelson et al. 2012). For clarity we define $M_{\mathrm{HSE}}$ to be the mass derived using $P=P_{\mathrm{th}}$ and $M_{\mathrm{tot}} \equiv M_{\mathrm{DM}}+$ $M_{\text {gas }}+M_{\text {star }}$. We define the bias owing to the assumption of HSE as $M_{\mathrm{HSE}} / M_{\text {tot }}$, which shows a weak increase in radius. Note that this definition differs from other approaches in the literature. Adopting $\tilde{M}_{\text {tot }} \propto \mathrm{d}\left(P_{\text {th }}+P_{\text {kin }}\right) / \mathrm{d} r$ results in a radially decreasing bias, presumably due to violation of spherical symmetry and non-stationary, clumpy accretion that is not accounted for in the derivation of $\tilde{M}_{\text {tot }}$ (see Appendix B).

Comparing $M_{\mathrm{HSE}}$ to the true mass inside a given radius, $M_{\text {HSE }}$ on average underestimates $M_{\text {tot }}$ by $20-25 \%$ within $R_{200}$ (BBPS1) and biases $f_{\text {gas }}$ accordingly high (dotted lines in Figure 11). This $M_{\mathrm{HSE}}$ bias is almost independent of cluster mass out to $R_{200}$ as can be seen by the fractional difference of $f_{\text {gas }}$ (lower panels of Figure 1). However, individual clusters can stray from this average deviation, since each cluster has a unique dynamical state and formation history. This is suggested by a scatter of $\sim 5 \%$ between the $25^{\text {th }}$ and $75^{\text {th }}$ percentiles of the complete distribution (BBPS1). For approaches that assume a constant $M_{\mathrm{HSE}}$ bias, the fractional difference of $f_{\text {gas,HSE }}$ would be accordingly shifted downwards (by the same factor in the lower panels of Figure 1 .

When we select a sample of the third most relaxed clusters, the correction factor will necessarily be smaller. We find that the bias on $f_{\text {gas }}$ for this sample is reduced to $15-20 \%$ within $R_{200}$. In a small sample of zoomed-cluster simulations that has been selected against major mergers the hydrostatic mass correction was found to be of the order $M_{\mathrm{HSE}} \sim 10-15 \%$ (Kravtsov et al. 2006). However, in both cases, we see that outside $R_{200}$, the hydrostatic bias rises steeply as $P_{\text {kin }} / P_{\text {th }}$ approaches large values and eventually reaches equipartition around $2 R_{200}$ (at $z=0$ ) with the exact equipartition radius depending on cluster mass.

\subsection{Clumping bias}

The emissivity of thermal bremsstrahlung scales with the square of the gas density. Hence the X-ray-inferred gas density profile is biased high by the presence of a clumped medium (Nagai \& Lau 2011; Roncarelli et al. 2013) and attains a bias of the square root of the density clumping factor, that we define by

$$
\tilde{\mathcal{C}}_{2, \rho}=\frac{\left\langle\rho^{2}\right\rangle}{\langle\rho\rangle^{2}} .
$$



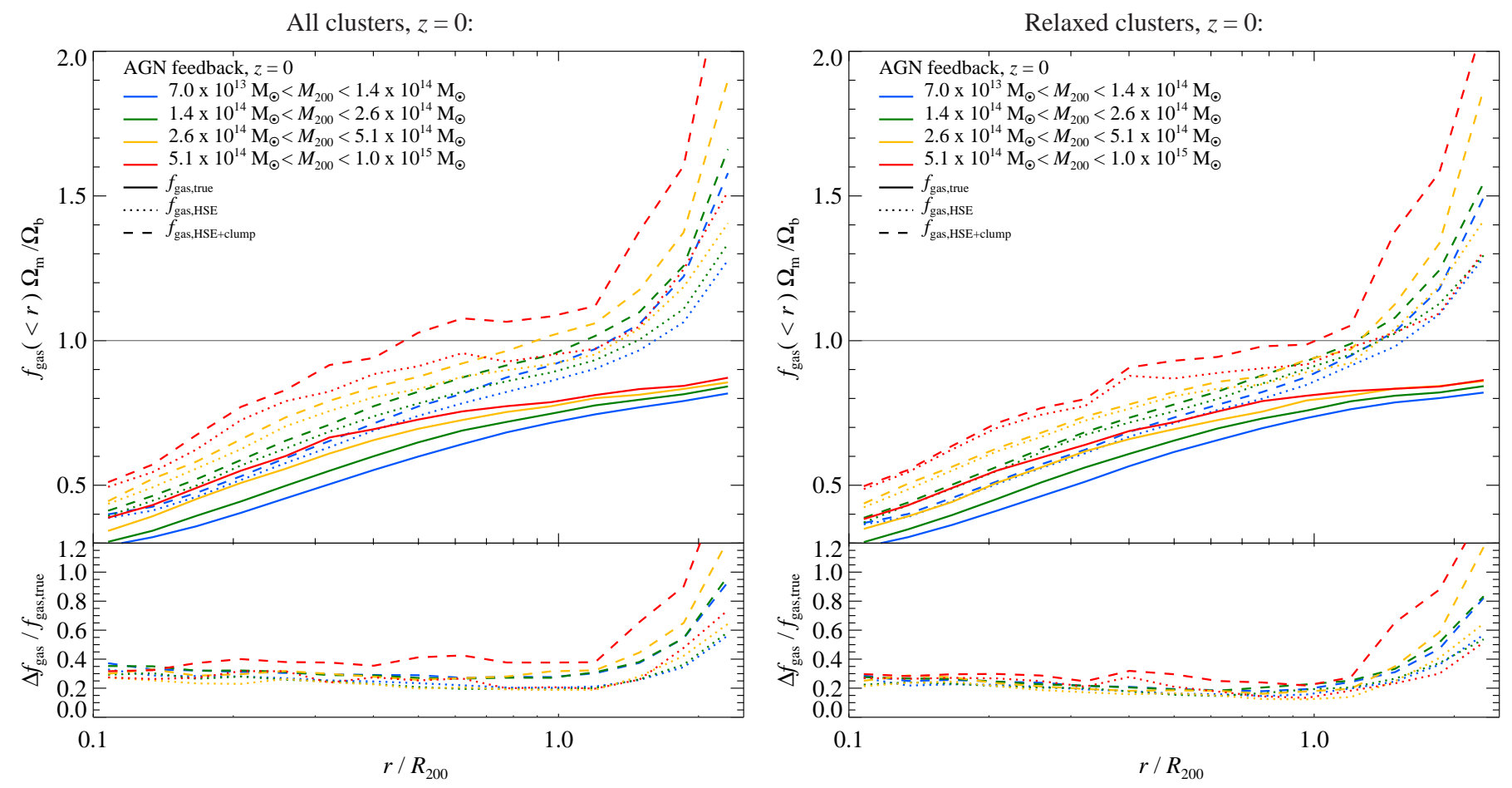

Figure 1. The assumption of hydrostatic equilibrium and of a smoothly distributed ICM introduces a bias in the measurement of $f_{\text {gas }}$. We show the median radial profiles of the gas mass fraction, $f_{\text {gas }}(<r) \equiv M_{\text {gas }}(<r) / M_{\text {tot }}(<r)$, normalized by the universal baryon fraction for different assumptions in calculating $M_{\text {gas }}$ and $M_{\text {tot }}$ as a function of cluster mass. We compare the true gas mass fraction, $f_{\text {gas,true }}$ (solid), a biased X-ray measurement of $f_{\text {gas }}$ due to the assumption of HSE, i.e., without accounting for kinetic pressure support, $f_{\text {gas.HSE }}$ (dotted), as well as a biased measurement of $f_{\text {gas }}$ due to the assumption of HSE and additionally neglecting density clumping, $f_{\text {gas.HSE+clump }}$ (dashed). The profiles in the left panel reflect the median of the distribution of all clusters and the right panel shows the median of a sub-sample of the third most relaxed clusters (with the lowest ratio of total kinetic-to-thermal energy, $K / U$ ). The bottom panels show the fractional difference from

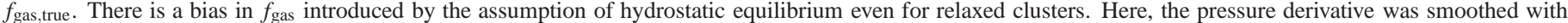
adjacent radial bins.

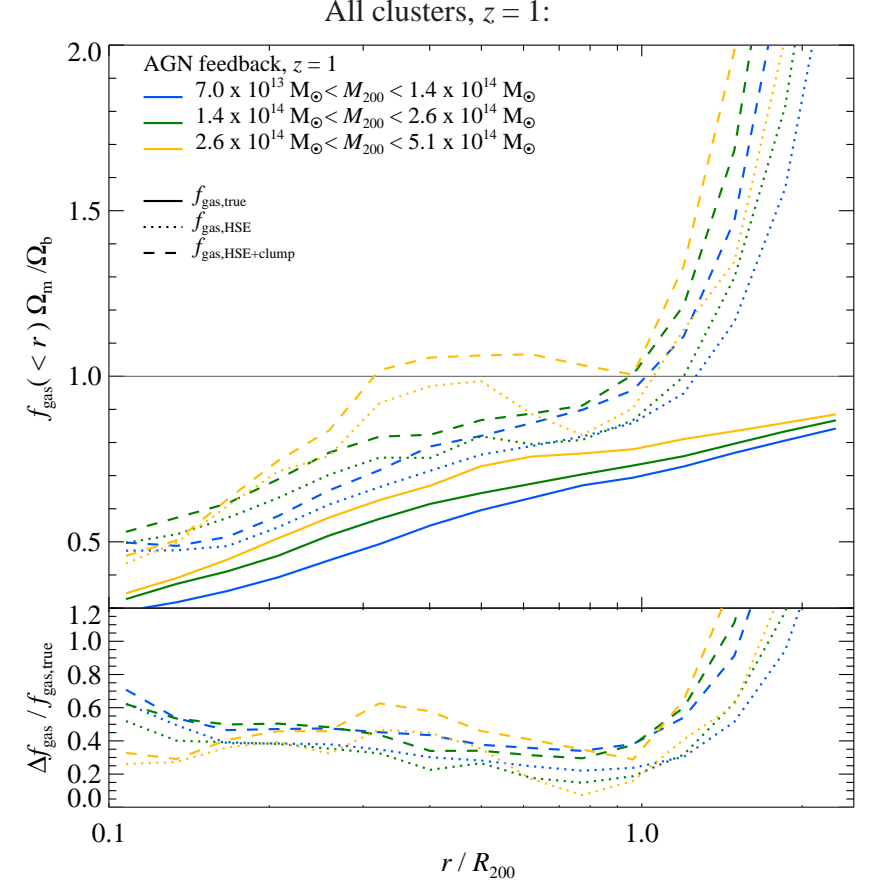

Figure 2. Same as in Figure 1 but at $z=1$ for fixed cluster mass bins (at $z=1$ there is no cluster with $M_{200}>5 \times 10^{14} \mathrm{M}_{\odot}$ in our simulations). While the intrinsic gas mass fraction shows no significant evolution with $z$, the assumption of hydrostatic equilibrium and of a smoothly distributed ICM introduces an increasing bias at $z=1$.
Averages are taken to be volume averages. We also define the SPH volume bias,

$$
\tilde{\mathcal{C}}_{0}=\langle 1\rangle=\frac{1}{V} \sum_{i} \frac{M_{i}}{\rho_{i}}=\frac{1}{V} \sum_{i} V_{i}
$$

that we expect to be identical to unity everywhere. While this is the case for small radii, the SPH volume bias deviates progressively for larger radii. It reaches a negative bias of $30 \%$ at $3 R_{200}(z=0)$, almost independent on the simulated physics and cluster masses, but not of redshift since it doubles at $z=1$ when radii are scaled to $R_{200}$ (BBPS4). To obtain unbiased estimates, we decided to redefine the clumping factor, yielding

$$
\mathcal{C}_{2, \rho}=\tilde{\mathcal{C}}_{2, \rho} \tilde{\mathcal{C}}_{0}
$$

where the multiplication with the volume bias factor, $\tilde{\mathcal{C}}_{0}$, has been chosen to cancel the SPH volume in the weighting. Inside $R_{200}$, the profile of $\sqrt{\mathcal{C}_{2, \rho}}$ increases moderately with radius but remains $<1.2$ for $r<R_{200}$ for all clusters. Outside $R_{200}$, it starts to rise sharply and reaches values of 10 at $r \simeq 3 R_{200}$ and $4 R_{200}$ for large clusters and groups, respectively, almost independent of the simulated physics (BBPS4). At each radius, there is a clear trend of an increasing clumping factor for larger clusters, which have on average a higher mass accretion rate, i.e., they are assembling at the current epoch and hence are dynamically younger.

Cold, dense gas clumps that are ram-pressure stripped from galaxies and find themselves in pressure equilibrium with the ambient hot ICM add an artificial bias to the density clumping factor. This bias needs to be accounted for when comparing 
to X-ray observations by employing, e.g., a temperature cut. Hence, in our calculations of the density clumping factor, we only consider SPH particles with $T>10^{6} \mathrm{~K}$ that can be observed by their X-ray emission.

To estimate the clumping bias of the enclosed gas mass fractions, we compute a clumping-biased density profile for each cluster by means of $\rho_{\text {clump }}(r)=\sqrt{\mathcal{C}_{2, \rho}(r)} \rho_{\text {true }}(r)$, and integrate this to obtain a clumping-biased gas mass profile,

$$
M_{\text {gas }, \text { clump }}(<r)=4 \pi \int_{0}^{r} \rho_{\text {clump }}\left(r^{\prime}\right) r^{\prime 2} d r^{\prime} .
$$

Here, we only asses the effect of clumping bias on $M_{\text {gas }}$. In principle, density clumping should also impact the determination of $M_{\text {tot }}$. However, there are different subtle effects that may partially compensate. Those include larger Poisson scatter in density profiles implied by clumping, which then have a noisier derivative, calling for some smoothing procedure which may bias the inferred masses. Also, the X-ray emissivity is enhanced by the presence of self-bound (dense) structures which increases the logarithmic derivative of the density and hence $M_{\text {tot }}$. Conversely, these dense substructures have a lower temperature. Fitting a single temperature spectrum to the data will then bias the inferred temperature and hence $M_{\text {tot }}$ low. Simulated X-ray observations are needed to carefully address these points that shall be subject to future work.

Clumping bias increases inferred values for $f_{\text {gas }}-$ similar to the $M_{\mathrm{HSE}}$ bias. In Figure 1, we show the effect of both biases that we denote by $f_{\text {gas,HSE+clump (dashed lines). As a re- }}$ sult of both biases, $f_{\text {gas }}$ realizes its universal value at considerably smaller radii in comparison to the true $f_{\text {gas }}$. In our sample of relaxed clusters, the universal value is reached outside $R_{200}$ whereas in our all-cluster sample, these high values are already obtained at $0.5 R_{200}$ for big clusters with $M_{200}>5 \times 10^{14} \mathrm{M}_{\odot}$. Interestingly, the biased values for $f_{\text {gas }}$ increase sharply outside the virial radius, mainly driven by the clumping bias for large systems and with a substantial contribution of $M_{\mathrm{HSE}}$ bias for smaller clusters. $f_{\text {gas }}$ is biased high by a factor of two at $2 R_{200}$ for large clusters and at $3 R_{200}$ for groups (see Figure 1 ).

What is the relative contribution of these measurement biases as a function of radius and cluster mass? In our all-cluster sample, clumping bias has a small effect at the cluster center but is continuously increasing towards $R_{200}$. At $R_{200}$, it approximately contributes half as much as the $M_{\mathrm{HSE}}$ bias to the overall bias with an absolute bias of $10-20 \%$, depending on cluster mass. In our relaxed cluster sample, the clumping bias remains even more subdominant in comparison to the $M_{\mathrm{HSE}}$ bias. However, in both cluster samples, the clumping bias increases dramatically beyond $R_{200}$ with an increasing bias with cluster mass.

Clusters form as a result of hierarchical merging of smaller systems. Hence the kinetic pressure support (BBPS1) and the density clumping (Nagai \& Lau 2011, BBPS4) increase with $z$ at fixed mass and so do the associated measurement biases of $f_{\text {gas }}$. This can be appreciated in Figure 2] which shows the radial profile of $f_{\text {gas }}$ at $z=1$. While the intrinsic gas mass fraction shows no significant evolution with $z$, the assumption of hydrostatic equilibrium and of neglecting the density clumping introduces an increasing bias at $z=1$ in comparison to the present epoch.

\subsection{Cluster sample variance}

It has been suggested that sample variance of clusters could possibly account for values of $f_{\text {gas }}$ larger than the cosmic mean, i.e., when a cluster experiences an ongoing merger or has closeby cluster companions. To address such questions, our large sample of clusters is ideal. It consists of 800 clusters with $M_{200}>1 \times 10^{14} \mathrm{M}_{\odot}$ and has 200 clusters with $k T_{\text {spec }}>2.5 \mathrm{keV}$ (corresponding to $M_{500}>2.15 \times 10^{14} \mathrm{M}_{\odot}$ ). In Figure 3] we compare the cluster-to-cluster scatter of $f_{\text {gas }}$ in a sample of all clusters and a sub-sample of the third most relaxed clusters (as defined by the third of all clusters with the lowest ratio of kinetic-to-thermal energy, $K / U$, within $R_{200}$, see Equations (1) and (2)). The sample variance of the true $f_{\text {gas }}$ decreases with increasing radius from a relative $1-\sigma$ scatter of $20 \%(10-15 \%)$ at $0.1 R_{200}$ for our sample of all (relaxed) clusters down to $\sim 5 \%$ at $R_{200}$. This demonstrates the smaller influence of AGN feedback on large scales especially for big clusters where the gravity of the DM potential starts to dominate. In contrast, the scatter of the biased quantity $f_{\text {gas,HSE }}$ remains fairly constant at a level of $\sigma_{f_{\text {gas }}} / f_{\text {gas }} \simeq 0.1-0.2$ (depending on the dynamical state and mass of clusters). Within $R_{200}$, the sample variance $f_{\text {gas }}$ due to the clumping bias is negligible, but it starts to rise steeply outside $R_{200}$, the innermost locations of accretion shocks (along filaments) that dissipate kinetic accretion energy and smooth the density distribution interior to this radius.

The $1-\sigma$ envelope of $f_{\text {gas, HSE+clump }}$ starts to rise dramatically for $r \gtrsim R_{200}$ for our all-cluster sample. This rise is shifted to somewhat larger radii for our sample of relaxed clusters (Figure 3). However, at $R_{200}$ we obtain an upper $1-\sigma$ percentile of $f_{\text {gas,HSE+clump }}$ for our massive clusters $\left(M_{200}>3 \times 10^{14} \mathrm{M}_{\odot}\right)$ that is $30 \%$ above the universal value for our all-cluster sample and only $10 \%$ for our subsample of relaxed clusters. Cluster variance alone remains unlikely to support values that are 1.6 times the cosmic mean - the measured value at $R_{200}$ in Perseus when accounting for a stellar mass fraction of $12 \%$ (Simionescu et al. 2011, even when accounting for the moderate increase of this number by $\sim 10 \%$ for the most massive clusters around $\left.M_{200} \sim 10^{15} \mathrm{M}_{\odot}\right)$. Moreover, clusters that are lying outside the $1-\sigma$ interval are either merging systems or are caught in a pre-merger state, properties that can easily be verified through optical or X-ray observations of the local environment of such a cluster (unless the merger axis is aligned with the line-of-sight, but such a configuration is rare and should be easily separable in a statistical sample of clusters).

\subsection{Angular variance of the mass distribution within a single cluster}

In nearby clusters, the large angular extent of clusters renders it impossible to cover the entire virial region of a cluster with a few X-ray pointings. Instead, a limited number of mosaic pointings along radial arms are being observed which opens the possibility of obtaining a biased answer for $f_{\text {gas }}$ if there is a considerable angular variance. Two effects may play a role. First, a large mass fraction is being accreted along filaments. These anisotropic ICM features associated with largescale structure environment suggest that the thermalization of the ICM occurs at smaller radii along overdense filamentary structures (that exert a greater ram pressure on the hot ICM) than along low-density void regions. Second, since there is a strong internal baryon-to-dark-matter density bias, a consequence of collisional-to-collisionless physics, this could in principle also lead to a significant angular variance depending on the differing efficiencies of gas thermalization and the relaxation processes of the DM component.

To quantify these considerations, in Figure 4 we show the profile of $M_{\text {gas }}, M_{\text {tot }}$, and $f_{\text {gas }}$ in angular cones centered on one 

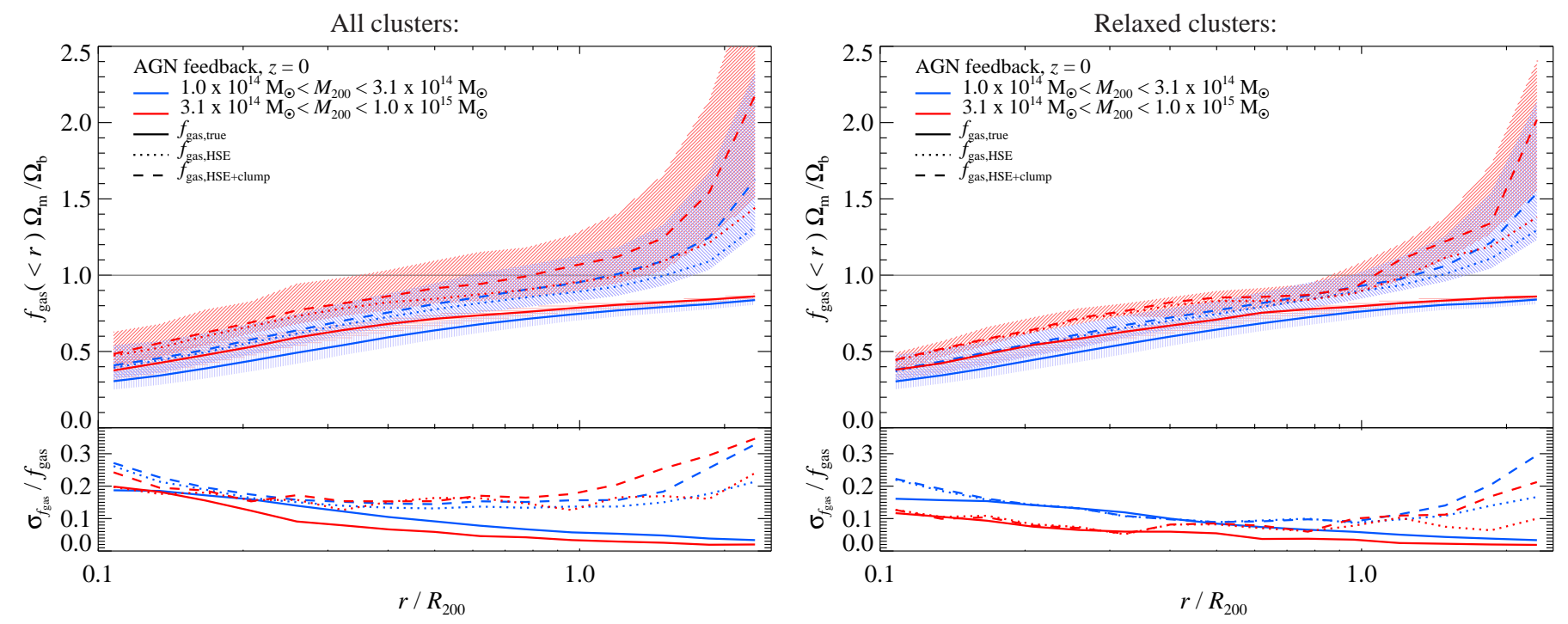

Figure 3. Cluster sample variance of $f_{\text {gas }}$. Upper panels: we show the gas mass fraction $f_{\text {gas }}$ for different mass intervals (lines indicate median values, shaded regions are bounded by percentiles such that they contain $68.3 \%$ of the probability, centered upon the median). Lower panels: we show the relative cluster-to-cluster scatter of the gas mass fraction, $\sigma_{f_{\mathrm{gas}}}$, that we define by the difference of the upper and lower $(1-\sigma)$ percentiles normalized by twice the median of $f_{\text {gas. }}$. Here, we compare radial profiles of the true gas mass fraction, $f_{\text {gas,true }}$ (solid), a biased X-ray measurement of $f_{\text {gas }}$ due to the assumption of HSE, i.e., without accounting for kinetic pressure support, $f_{\text {gas,HSE }}$ (dotted), as well as a biased measurement of $f_{\text {gas }}$ due to the assumption of HSE and additionally neglecting density clumping, $f_{\text {gas, HSE+clump }}$ (dashed). The profiles in the left panel include all clusters and the right panel shows the median of a sub-sample of the third most relaxed clusters (with the lowest ratio of total kinetic-to-thermal energy, $K / U$ ). While the scatter of $f_{\text {gas,true }}$ decreases for larger radii, the scatter of the biased $f_{\text {gas,HSE+clump }}$ remains fairly constant at a level of $\sigma_{f_{\text {gas }}} / f_{\text {gas }} \simeq 0.1-0.2$ (depending on the dynamical state and cluster mass) within $R_{200}$ and even starts to increase for larger radii due to the strong clumping term. (We do not show the shaded regions of $f_{\text {gas,HSE }}$ for visual clarity, but show this information in the lower panels.)
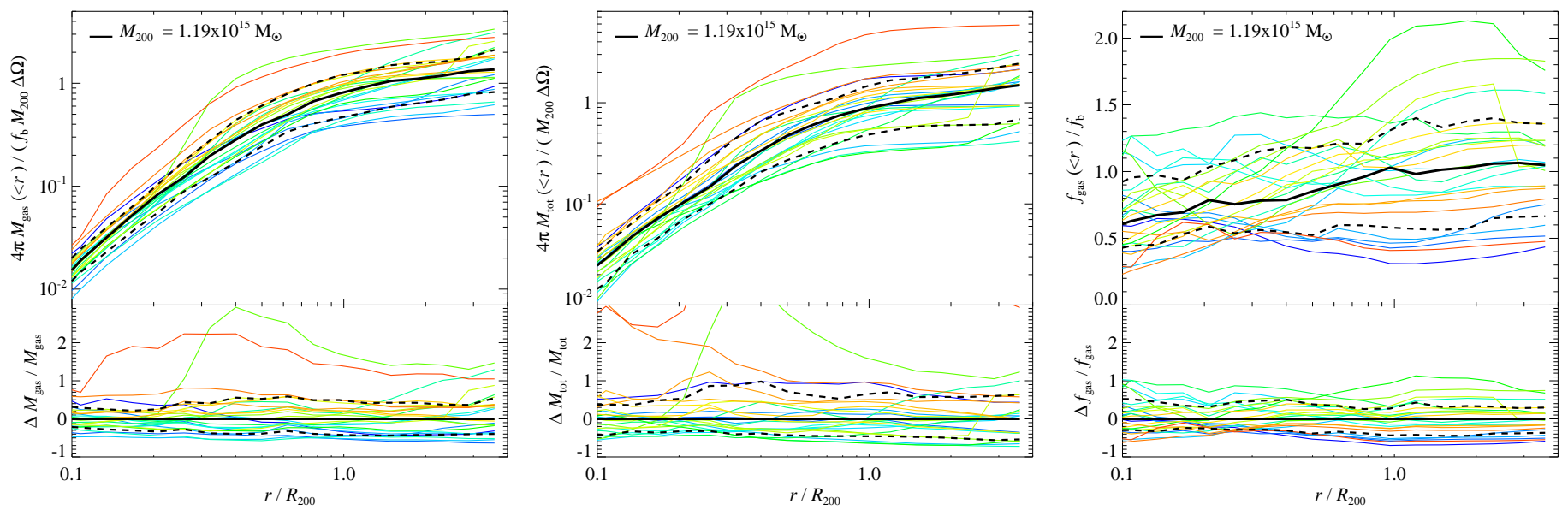

Figure 4. Upper panels: we show the profiles of $M_{\text {gas }}$ (left), $M_{\text {tot }}$ (middle), and $f_{\text {gas }}$ (right) in 48 angular HEALPix cones (which subtend an equal area pixelization on the sphere), centered on one cluster of mass $M_{200} \approx 1.2 \times 10^{15} \mathrm{M}_{\odot}$. (Note that we only show every other profile for visual purposes.) We overplot the median (solid black) as well as the upper and lower $(1-\sigma)$ percentiles (dotted black). Lower panels: we show the variations of $M_{\mathrm{gas}}, M_{\mathrm{tot}}$, and $f_{\mathrm{gas}}$ in the different cones relative to their corresponding median. Note the different scalings at the $y$-axis (logarithmic for the mass profiles, linear scale for $f_{\text {gas }}$ ). There is considerable scatter as well as the presence of a few enormous outliers in the $M_{\text {gas }}$ and $M_{\text {tot }}$ cones - depending on the angular direction - which is reduced for $f_{\text {gas }}$. This implies spatial correlations of the DM and gas distribution through self-bound substructures that are still holding on to (some) of their gas.

massive cluster of $M_{200} \approx 1.2 \times 10^{15} \mathrm{M}_{\odot}$. We chose the angular directions of these 48 cones such that they subtend an equal area pixelization on the sphere (following the HEALPix scheme with $N_{\text {side }}=2$; Górski et al. 2005). The number of cones is a compromise between obtaining high-enough angular resolution to break the angular smoothing that is necessarily present in sphericalized profiles and to resolve individual substructures and cosmic filaments in these cones. At the same time, we aim at minimizing scatter due to our finite number of particles. To calculate $M_{\mathrm{tot}}$, we sum up the mass distribution of the gaseous, stellar, and DM components in those cones》

We observe considerable scatter in all three distributions, $M_{\mathrm{gas}}, M_{\mathrm{tot}}$, and $f_{\mathrm{gas}}$, depending on the angular direction. There are a few enormous outliers in the $M_{\mathrm{gas}}$ and $M_{\text {tot }}$ cones, deviating upwards up to a factor of 3 from the median (shown with solid black) in the case of $M_{\text {gas }}$ and even beyond for $M_{\text {tot }}$. The right-most panel in Figure 4 shows that in the most extreme cases, the cone profiles of $f_{\text {gas }}$ can be biased high by a factor

\footnotetext{
${ }^{1}$ For the gas component, we sort the volume fraction of an SPH smoothing kernel into the corresponding radial bins, but neglect the extension of the smoothing kernel in angular directions for performance reasons (see Appendix C
} 

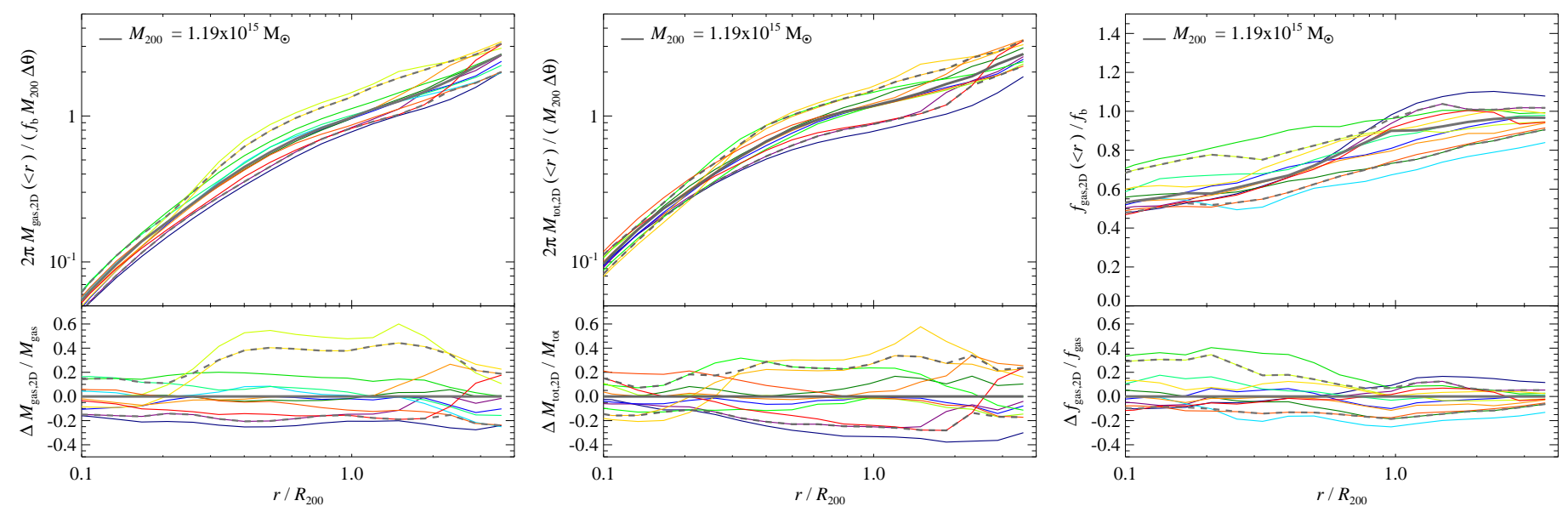

Figure 5. Upper panels: we show the projected (cylindrical) profiles of $M_{\text {gas }}$ (left), $M_{\text {tot }}$ (middle), and $f_{\text {gas }}$ (right) in 12 equally large angular sectors, centered on one cluster of mass $M_{200} \approx 1.2 \times 10^{15} \mathrm{M}_{\odot}$. We overplot the median (solid black) as well as the upper and lower $(1-\sigma)$ percentiles (dashed black). Lower panels: we show the variations of $M_{\text {gas }}, M_{\text {tot }}$, and $f_{\text {gas }}$ in the different sectors relative to their corresponding median. Note the different scalings at the $y$-axis (logarithmic for the mass profiles, linear scale for $f_{\text {gas }}$ ). In comparison to the $3 \mathrm{D}$ cones, there is less scatter and less dramatic outliers in the projected sector distribution of $M_{\text {gas }}, M_{\text {tot }}$, and $f_{\text {gas }}$.

of two in such a massive cluster with $M_{200} \sim 10^{15} \mathrm{M}_{\odot}$, potentially providing an explanation of the boosted values of $f_{\text {gas }}$ reported in the north-western arm in Perseus (Simionescu et al. 2011). Care should be taken when interpreting these $f_{\text {gas }}$ values in cones, since projection effects may lower these values. However, as demonstrated in Figure 1, hydrostatic mass bias as well as density clumping may (partially) compensate for these projection effects.

Only in the $M_{\text {tot }}$ cones, those outliers can be seen down to small radii $r \lesssim 0.1 R_{200}$ which is expected as DM substructure only obeys collisionless physics that allows substructures to penetrate deep into the halo without too much affecting the dense central subhalo structures by tidal stripping. In contrast, the gaseous content has been removed by means of shocks or ram-pressure stripping at larger radii, lowering the variance of $M_{\text {gas }}$ at smaller radii (see Figure 4). These large outliers are absent in the distribution of $f_{\text {gas }}$ cones, which implies a smaller variance (dashed black). This suggests that these outliers are caused by self-bound substructures that are still holding on to (some) of their gas at larger cluster radii, i.e., there exists a tight spatial correlation of the DM and gas distribution (see BBPS4 for a detailed analysis).

Line-of-sight projection smoothes out the largest angular variations in the 3D distribution. We calculate projected (cylindrical) mass distributions according to

$$
\begin{gathered}
\Sigma\left(r_{\perp}\right)=\int_{0}^{L} \rho\left(r_{\perp}, l\right) d l, \\
M_{2 \mathrm{D}}\left(<r_{\perp}\right)=2 \pi \int_{0}^{r_{\perp}} \Sigma\left(r_{\perp}^{\prime}\right) r_{\perp}^{\prime} d r_{\perp}^{\prime},
\end{gathered}
$$

where $L=165 h^{-1} \mathrm{Mpc}$ denotes the length of the simulation box. Before projecting, we smooth the discrete stellar and DM masses by distributing their individual masses according to an SPH-type smoothing kernel that extends up to the nearest $48^{\text {th }}$ neighbouring particle (of the same type). In Figure 5, we show the resulting projected profiles of $M_{\text {gas }}, M_{\text {tot }}$, and $f_{\text {gas }}$ in 12 equally large angular sectors, centered on the same massive cluster as studied in Figure 4 In comparison to the 3D cones, the scatter is smaller and there are less dramatic outliers in the projected sector distribution of $M_{\text {gas }}, M_{\text {tot }}$, and $f_{\text {gas }}$. Unlike the $3 \mathrm{D}$ case, the largest 2D outlier sector in this cluster does not exceed far above the cosmic mean of $f_{\text {gas }}$ within $R_{200}$.

In Appendix D, we show that the variance of projected sectors of $M_{\text {gas }}$ and $f_{\text {gas }}$ depends only very weakly on the number of sector elements, hence justifying our choice of 12 elements. Instead, the scatter of the X-ray luminosity strongly depends on the number of sector elements, especially in SPH simulations (see Figure 4 of Vazza et al.2011b). Presumably, this increased scatter solely derives from the increased clumping factor with radius. The increased scatter in the SPH simulations in comparison to AMR simulations especially for $r<R_{200}$ Vazza et al. 2011b) may stem from an incomplete clump removal.

\subsection{Variation of the mass distribution with angle across cluster samples}

In order to obtain statistically valid answers on how this scatter across different cones varies among our cluster sample, we first compute the variance of the profiles of $M_{\text {gas }}, M_{\text {tot }}$, and $f_{\text {gas }}$ across 48 angular cones (dashed lines in Figure 4) for all clusters. Since the distribution within a single cluster is nonGaussian and positively skewed (by the presence of substructures), we define the angular variance of $M_{\mathrm{gas}}, \sigma_{M_{\mathrm{gas}}}$, by the difference of the upper and lower $1-\sigma$ percentiles, normalized by twice the median of $M_{\text {gas }}$. In the top panels of Figure 6 we show the mean of $\sigma_{M_{\text {gas }}}$ across our cluster sample (color coded by cluster mass). The same quantity is being calculated for $M_{\text {tot }}$ and $f_{\text {gas }}$ (lower panels of Figure 6) across a sub-sample of the third most relaxed clusters (left) and across all clusters (middle). Also shown is the mean of the angular variances $\sigma_{M_{\text {gas }}}$, $\sigma_{M_{\mathrm{tot}}}$, and $\sigma_{f_{\mathrm{gas}}}$ for the projected 2D maps (right).

As shown in Figure 6, there is an upturn of the raw 3D variances (dashed) visible at small radii and in particular for galaxy groups. This is caused by the noisy distribution of a comparably small number of SPH particles in the central cluster regions in combination with the smaller radial bin size of our logarithmic equally-spaced grid towards the center, when distributing the particle masses among the angular cones. As a result of the mutual repulsive hydrodynamical forces of neighboring SPH particles, they maintain a sub-Poissonian distribution with repulsive short distance correlations on scales of order the corresponding inter-particle separations. The full characterization of such a distribution with dynamical evolution of SPH is not possible in terms of known distributions. Hence we decided to cap 
Relaxed clusters, 3D:
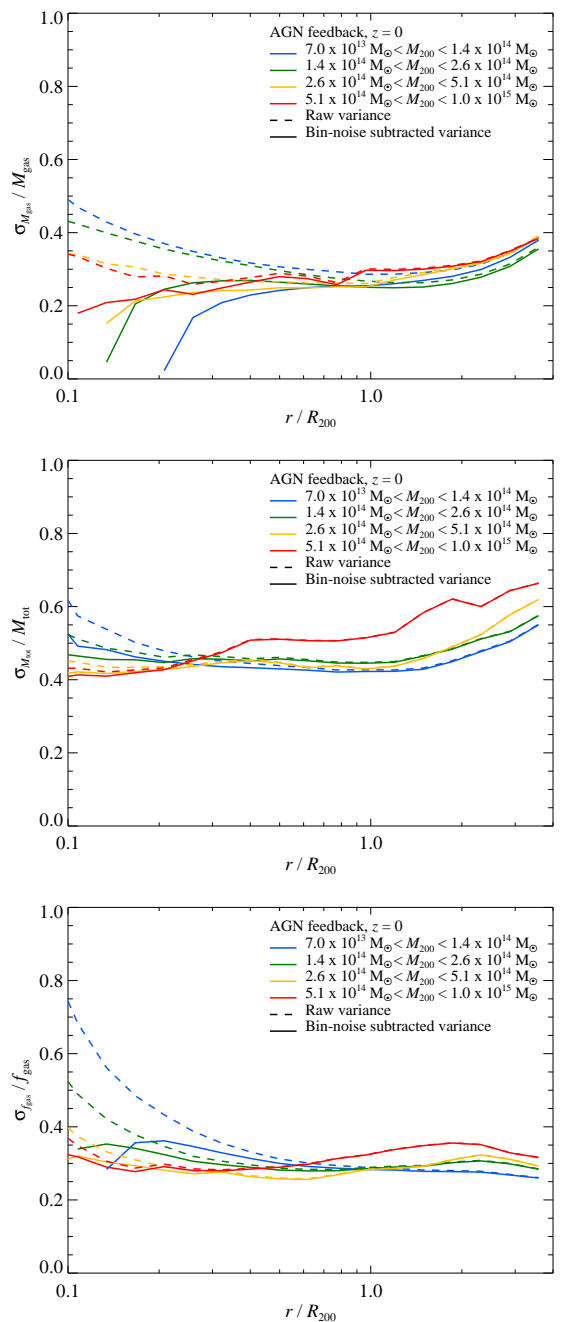

All clusters, 3D:
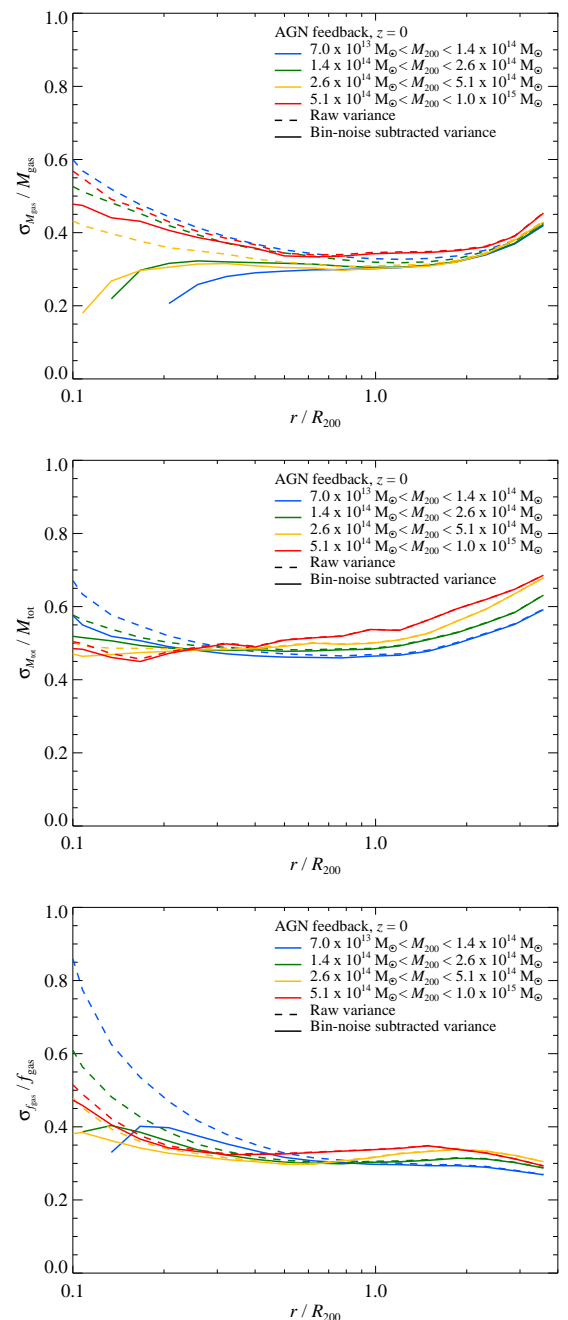

All clusters, 2D:
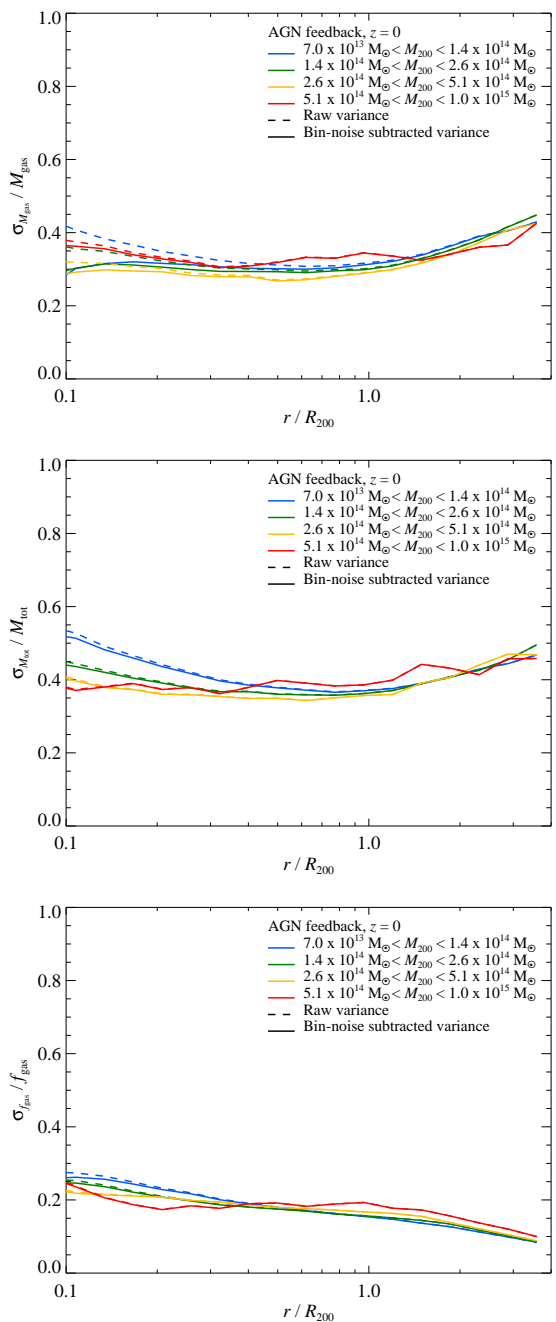

Figure 6. Top panels: we compute the variance of the $M_{\text {gas }}$ profiles across 48 angular 3D cones using the HEALPix scheme (dashed lines in Figure 4) and across 2D angular sectors (dashed lines in Figure 5 for each cluster. Here we show the mean of the 3D cone variances across a sub-sample of the third most relaxed clusters with the lowest ratio of kinetic-to-thermal energy, $K / U$ (left) and across all clusters (middle). This is contrasted to the mean of the $2 \mathrm{D}$ sector variance (right). We define the angular variance of $M_{\mathrm{gas}}, \sigma_{M_{\mathrm{gas}}}$, by the difference of the upper and lower $(1-\sigma)$ percentiles normalized by twice the median of $M_{\mathrm{gas}}$. We compare the measured variance (dashed) to our estimate of the intrinsic angular variance (solid) where a Poisson noise term due to the finite particle number within the cones (respectively sectors) has been subtracted in quadrature. Note that in the 3D case this over-corrects the intrinsic variance at small radii as the Poisson noise estimate caps the noise contribution owing to the true sub-Poissonian distribution of SPH particles, which get rearranged as a result of the mutual repulsive hydrodynamical forces. Middle and bottom panels: same as above, but for $M_{\text {tot }}$ and $f_{\text {gas }}$, respectively, where $M_{\text {tot }}$ is calculated by summing up the mass distribution of the gaseous, stellar, and DM components in those cones/sectors. Different colors correspond to different cluster mass ranges (indicated in the legends). Due to its collisional nature, gas is able to dissipate part of its kinetic accretion energy more efficiently than DM, thereby reducing its angular variance, in particular for relaxed systems (on the right). This is in contrast to the collisionless DM component (dominating the total mass) which shows a larger angular variance of its mass distribution.

this numerical noise contribution by treating it as Poissonian and subtract a Poissonian noise term in quadrature from the raw $3 \mathrm{D}$ angular variance. We caution that this over-corrects the intrinsic variance at small radii, causing an artificial decrease of the bin-noise subtracted variance (solid). We emphasize that our focus here is on large scales with $r \gtrsim(0.2-0.5) R_{200}$ (where the range corresponds to large and small clusters, respectively). Those scales are not subject to this numerical limitation. Hence in order to improve upon our analysis in the central regions, an increase in numerical resolution would be needed. The $2 \mathrm{D}$ angular variances in the central regions are only little affected by the sub-Poissonian variance due to the sufficiently large number of particles that are projected onto the central regions.

Inside $R_{200}$, the 3D angular variance of $M_{\text {gas }}$ does not strongly depend on mass and reaches values around $30 \%$ at $R_{200}$ with a moderate increase at larger radii. In contrast, the
3D angular variance of $M_{\text {tot }}$ is steadily increasing and reaches values around $45-50 \%$, depending on cluster mass (with the upper range indicating larger clusters that are on average dynamically younger). These values are only moderately reduced by $\simeq 5$ percentage points for our relaxed cluster sample. The different 3D angular variances of $M_{\mathrm{gas}}$ and $M_{\mathrm{tot}}$ can be understood by the collisional nature of the gas that is able to dissipate part of its kinetic accretion energy more efficiently (through shocks and ram-pressure stripping of the gaseous contents of substructures), thereby reducing its $3 \mathrm{D}$ angular variance. This is in contrast to the collisionless DM component (dominating the total mass) which shows a larger 3D angular variance of its mass distribution.

Interestingly, the Poisson noise-corrected 3D angular variance of $f_{\text {gas }}$ remains almost constant with radius at the level of $30-35 \%$ not only within the clusters but also for $r>R_{200}$. 

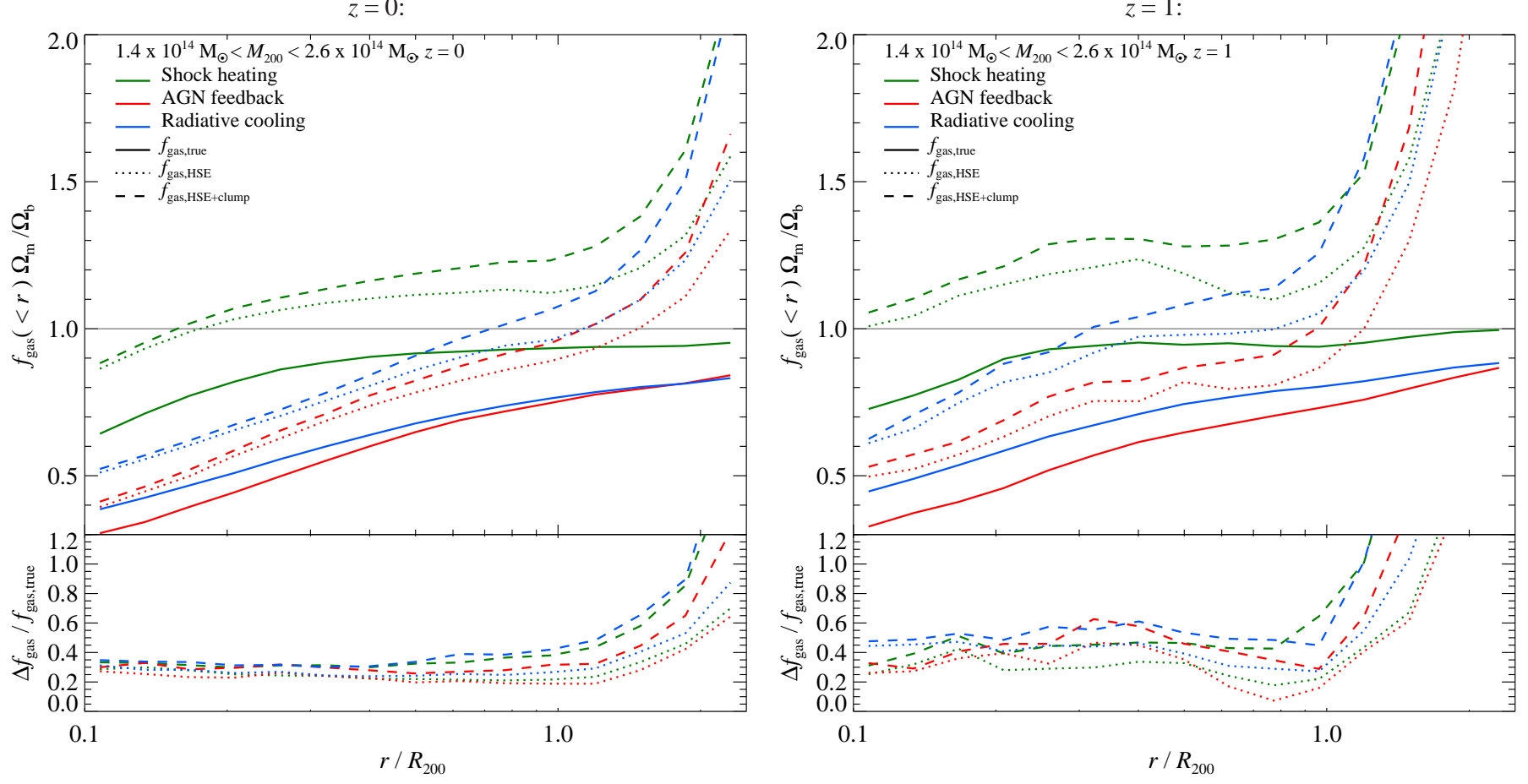

Figure 7. Different levels of sophistication of the simulated physics of the ICM imply different profiles for $f_{\text {gas }}$. In the top panels, we show the true gas mass fraction $f_{\text {gas }}(<r)$ (solid), $f_{\text {gas } \operatorname{HSE}}(<r)$ (dotted), and $f_{\text {gas HSE+clump }}(<r)$ (dashed), all normalized by the universal baryon fraction for different physics models at two redshifts, $z=0$ (left) and $z=1$ (right). In the bottom panels, we show the relative differences with respect to the true $f_{\text {gas }}$. There is little redshift evolution between the biases at $z=0$ and $z=1$, except for large radius at which these biases increase dramatically and which moves to smaller radii for $z=1$. The HSE bias of the gas mass fraction, $f_{\text {gas.HSE}}$, depends less on the simulated physics, whereas the $f_{\text {gas }}$ bias that additionally includes clumping, $f_{\text {gas.HSE+clump }}$, is marginally stronger in the $s h o c k$ heating and radiative cooling models in comparison to the AGN feedback simulations.

Furthermore, $\sigma_{f_{\text {gas }}} / f_{\text {gas }}$ depends very weakly on cluster mass. Both of these properties can be understood by the fact that at these large radii, most of the accreting substructures have not yet passed the accretion shock nor are they affected by rampressure stripping as they still move through the dilute warmhot inter-galactic medium. Hence these self-bound substructures are still holding on to their gas, implying large spatial correlations of the DM and gas distribution (see also Figure 4 and BBPS4).

The most striking feature of all our Poisson noise-corrected $3 \mathrm{D}$ angular variances is the rather constant behavior with radius, in particular for the 3D angular variances of $M_{\text {gas }}$ and $f_{\text {gas }}$, as well as the moderate radial increase of that of $M_{\text {tot }}$. In principle, the mass distribution in cones allows for finer sampling of the cluster shape beyond the moment-of-inertia tensor and is sensitive to higher-order moments - a measure that would be similar to density clumping for sufficiently high multipoles. In practice, this is limited by the Poisson noise due to our finite particle resolution. Moreover in Figure 6 we concentrated on the radial profiles of the second moment of the 3D angular variance which is intimately related but not identical to cluster ellipticities as obtained through eigenvalues of the moment-ofinertia tensor as a function of radius (BBPS1). The ellipticity of the gas density also shows a constant behavior inside $R_{200}$ while it is rising at larger radii. In contrast, the DM ellipticity shows already a moderate increase with radius which only starts to rise more steeply outside $R_{200}$ (BBPS1) - very similar to our findings for the $3 \mathrm{D}$ angular variance of $M_{\text {tot }}$ that is dominated by DM.

When averaged over sufficiently large scales, i.e. at very large radii, $f_{\text {gas }}$ is expected to assume the cosmic mean and the
3D angular variance $\sigma_{f_{\text {gas }}}$ should approach zero. In our case, the outermost radial bin is centered on $R_{i}=3.6 R_{200}$ and has a radial extend of $\Delta R_{i}=0.8 R_{200}$. The volume associated with that cone bin is $V_{\text {cone }, i}=\Delta R_{i} R_{i}^{2} 4 \pi / 48=(2.9 \mathrm{Mpc})^{3}$ for a $10^{15} \mathrm{M}_{\odot}$ cluster with $R_{200}=2.1 \mathrm{Mpc}$. This volume is still small in comparison to the scale associated with the root mean square variance of linear theory in spheres of radius $8 \mathrm{Mpc} / h, \sigma_{8}=0.8$. In fact, the $2 \mathrm{D}$ angular variance $\sigma_{f_{\mathrm{gas}}}$ shows the expected downturn for radii $r \gtrsim 2 R_{200}$. The volume associated with the outermost $2 \mathrm{D}$ radial bin of a cylindrical projection length of $L=165 \mathrm{~h}^{-1} \mathrm{Mpc}$ is $V_{\text {sector }, i}=L R_{200}^{2}\left(x_{i}^{2}-x_{i-1}^{2}\right) \pi / 12=(11.5 \mathrm{Mpc})^{3}$ for a $10^{15} \mathrm{M}_{\odot}$ cluster and $\left(x_{i}, x_{i-1}\right)=(4,3.2)$. This volume is 60 times larger than for the corresponding $3 \mathrm{D}$ case, but still somewhat smaller than the volume of a sphere with radius $8 \mathrm{Mpc} / h$. Why do we then observe a decline in the 2D angular variance? This is because the line of sight projection averages over many uncorrelated patches on scales $\gg 8 \mathrm{Mpc} / h$, which draws $\sigma_{f_{\text {gas }}}$ down for larger radii and brings $f_{\text {gas }}$ back to the cosmic mean. In the $3 \mathrm{D}$ case, the considerably smaller volume and the inherent radial correlations of the gas and DM distributions on these scales (as quantified in BBPS4) explain the flat radial profile of $\sigma_{f_{\text {gas }}}$ out to $4 R_{200}$.

All angular variances of $M_{\text {gas }}$ and $M_{\text {tot }}$ (2D and 3D) show an increase at the largest radii $\left(r \gtrsim 2 R_{200}\right)$. This reflects the increase of the root mean square variance, $\sigma_{R}^{2}=\left\langle\delta^{2}(\boldsymbol{x})\right\rangle=$ $\left\langle(\delta M / M)^{2}\right\rangle$, on these scales due to the dominance of the twohalo term in halo-mass cross-correlation, which corresponds to the mass autocorrelation function with a mass-dependent offset in amplitude known as the halo bias factor Havashi \& White 2008). 


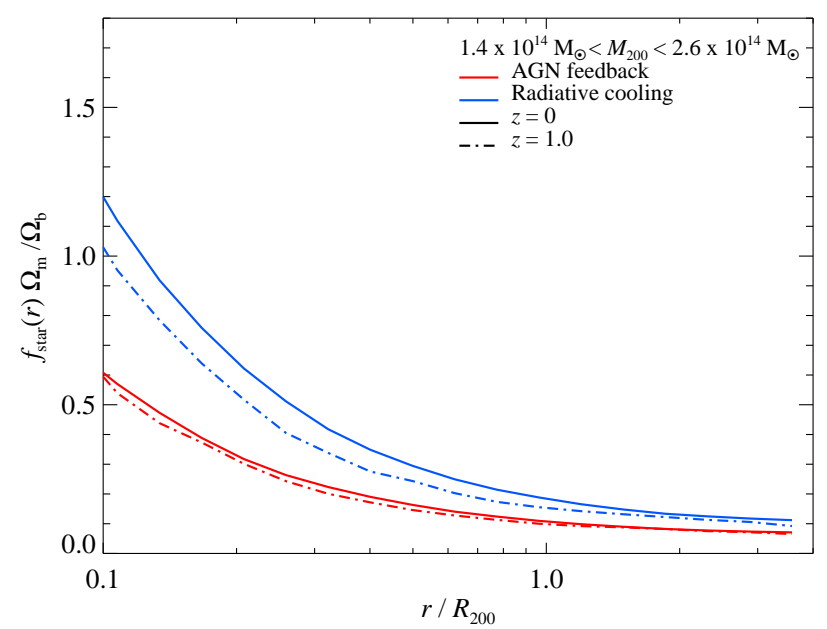

Figure 8. Whether or not we include AGN feedback does affect the median profile of the stellar mass fraction, $f_{\text {star }}$. We show $f_{\text {star }}(<r) \equiv M_{\text {star }}(<$ $r) / M_{\text {tot }}(<r)$ normalized by the universal baryon fraction at $z=0$ (solid) and $z=1$ (dash-dotted). While ongoing cooling increases the central stellar mass fraction to unphysical high values in our model with cooling and star formation-only, AGN feedback is able to self-regulate the core region and to suppress the forming cooling flow.

\section{GAS AND STELLAR MASS FRACTIONS IN COSMOLOGICAL} SIMULATIONS

Here, we present results on how $f_{\text {gas }}$ varies with simulated physics, radius, cluster mass, and redshift. These results are summarized in Table 1 and 2 .

\subsection{How different physics determines $f_{\text {gas }}$ and $f_{\text {star }}$}

Even though we believe that our AGN feedback model represents the closest approximation to reality, it is important to compare it to simpler physics models, which have been extensively studied in earlier literature. Moreover, there is considerable uncertainty about the correct physics underlying AGN feedback so that trends relative to our simpler models can be very instructive. Hence, we compare how different physics models impact the radial profiles of $f_{\text {gas }}$ (Figure 7) and $f_{\text {star }}$ (Figure 8). This is supplemented by a figure demonstrating the cluster mass dependence of $f_{\text {gas }}$ and $f_{\text {star }}$ within $R_{500}$ and $R_{200}$ for differently simulated physics (Figure 9 ).

In our shock heating-only model, $f_{\text {gas }}$ approaches a value of $0.93 \pm 0.03$ in units of $f_{c}$ at $r \sim R_{200}$ (which is reduced by one percentage point for larger clusters). Outside the virial radius, $f_{\text {gas }}$ is continuously rising to asymptote to the cosmic mean only at $4 R_{200}$. This may be partially due to a transfer of energy from DM to the gas during group/cluster mergers. In an equal mass collision, SPH simulations show that the DM can transfer up to $10 \%$ of its energy, and even a 10:1 merger may be able to reduce the DM energy by a couple of percent $\mathrm{McC}$ arthy et al. 2007). There is also the possibility that some of this gas deficiency may be related to the SPH technique that we use here. There appears to be a discrepancy to adaptive mesh-refinement simulations that find higher values of $f_{b} \approx 0.97 \pm 0.03$ within $R_{\text {vir }}$ (Kravtsov et al. 2005, see also Section 4.5, for a detailed discussion). At small radii, the shock-heated ICM provides pressure that causes the inner slope of the gas density profile to be rather flat, while the collisionless DM exhibits a cusp (e.g., Gao et al. 2012). As a result, this causes a decreasing gas fraction inside the DM scaling radius, i.e., for $r \lesssim 0.2 R_{200}$.

Our models with AGN feedback and radiative cooling have similar values of $f_{\text {gas }}$ at $z=0$, in particular for $r \gtrsim R_{500}$. How- ever, in the AGN feedback model, $f_{\text {gas }}$ only marginally evolves with $z$, while it increases for higher $z$ in the case of radiative cooling and star formation. This also manifests itself in the constant evolution of the $f_{\text {star }}$ profile in the AGN feedback model. In contrast, the stellar mass is increasing with time in the radiative model without AGN feedback due to the well-known overcooling problem in cosmological simulations of galaxy clusters if cooling is not counteracted by any feedback process (e.g., Borgani \& Kravtsov 2009).

For $r \gtrsim R_{200}$, the values of $f_{\text {gas }}$ in different radiative models (as well as for different redshifts) approach each other. However, in comparison to our shock heating-only model, there is still a gas mass deficit of $10 \%$ at $4 R_{200}$. In the raditive cooling model, the stellar mass fraction makes up for this deficit (Figure 8) while in the case of AGN feedback, stellar mass fractions are reduced by a factor of $\sim 2$ in comparison to our radiative cooling model. (Note that $f_{\text {star }}$ in our radiative cooling models depends on numerical resolution as this model is physically not converged.) The remaining gas mass has been pushed to even larger radii through the action of AGN feedback (McCarthy et al. 2011). Interestingly, these lower values for $f_{\text {gas }}$ in our radiative models in comparison to our non-radiative models imply a temperature increase in the outer parts (e.g., Figure 4 of Fabjan et al. 2010) that is necessary to balance the gravitational cluster potential.

At $z=0$, all our models show approximately a similar relative $M_{\mathrm{HSE}}$ bias (left bottom panel of Figure77) while the relative differences with respect to the true $f_{\text {gas }}$ are larger at $z=1$ (right bottom panel of Figure 77. However, there are some notable differences. At large radii, the radiative cooling model exhibits the largest bias, while our AGN feedback model shows the lowest bias. This is presumably due to AGN outflows depositing heat at these large radii (early on) which manifests itself in an increased pressure in comparison to the radiative cooling model (Battaglia et al. 2010). This increased thermal pressure moves the average location of accretion shocks to larger radii (since these form at the location where ram pressure due to infalling gas balances the thermal pressure of the ICM), which causes a deceleration of the accreting material already further out, and hence slightly decreases the average kinetic pressure support in the AGN feedback model. Hence, the bias corrections shown in Figure 1 are conservative given that the AGN feedback model shows an overall lower bias in comparison to the other models.

At $z=0$, the bias due to the hydrostatic equilibrium assumption and as a result of neglecting clumping ( $\left.f_{\text {gas,HSE+clump }}\right)$ is rather constant inside $R_{200}$ and shows values around $30 \%$. At higher redshifts, $z=1, f_{\text {gas,HSE+clump }}$ increases to a level that fluctuates around 50\%. Outside the virial radius, the $f_{\text {gas,HSE+clump }}$ bias increases sharply, albeit at a slightly slower rate in our AGN feedback model for reasons explained above. The location of the sharp increase in the bias moves from $\sim 2 R_{200}$ (at $z=0$ ) to $\sim R_{200}$ (at $z=1$ ). This is mostly due to the definition of $R_{200}$ and can be approximately aligned by changing the definition to $R_{200 m}$, which compares the average interior density to the mean mass density rather than the critical density of the Universe (Figure 21 of BBPS1).

\subsection{Gas and stellar mass fractions across cluster masses}

How do the gas and stellar mass fractions, $f_{\text {gas }}$ and $f_{\text {star }}$, as well as the sum of both, the baryon mass fraction, $f_{b}$, vary with cluster mass? In Figure 9 we show $f_{\text {gas }}$ and $f_{\text {star }}$ for our 3 different physics models as a function of cluster mass. As discussed in Section 4.1, our model that only accounts for shock 

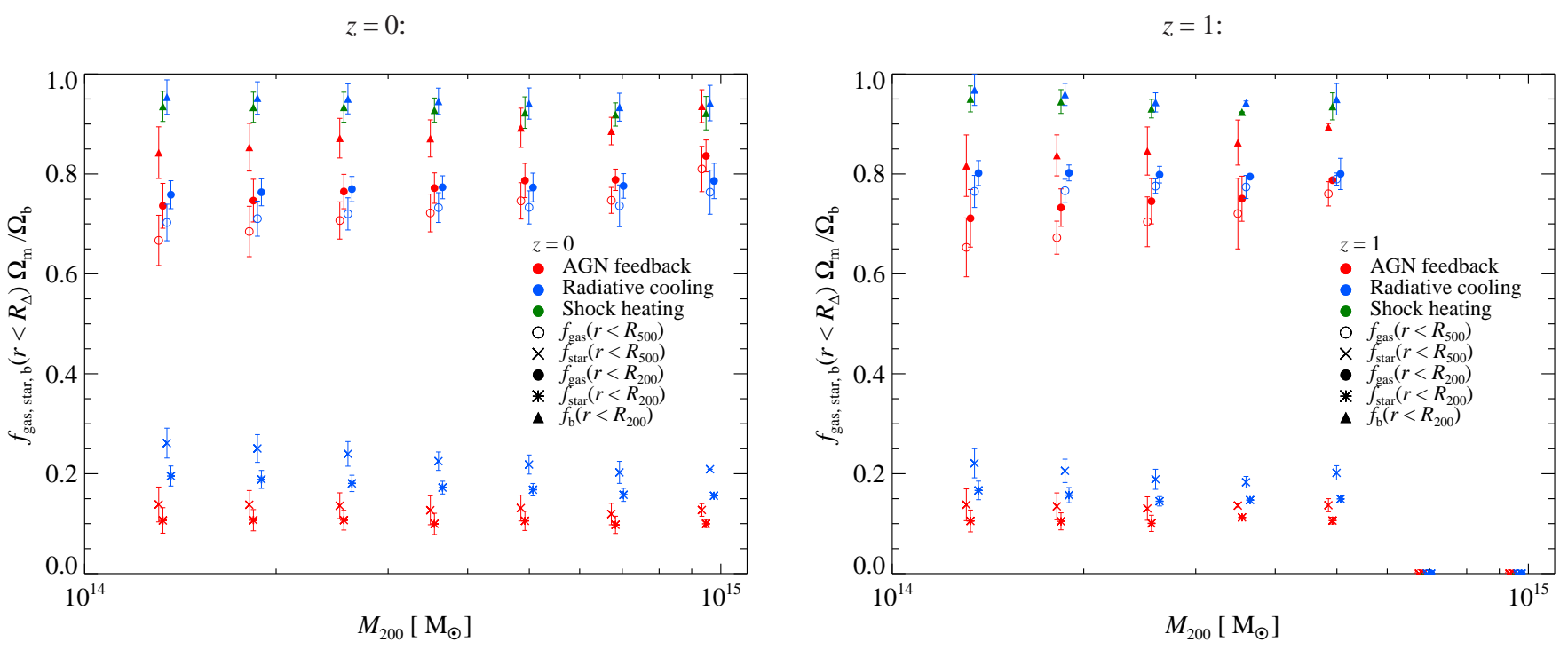

Figure 9. We show gas, stellar, and baryon mass fractions, $f_{\text {gas }}(<r), f_{\text {star }}(<r)$, and $f_{b}(<r)$, (mean and standard deviations), computed within a fixed radius $\left(R_{500}\right.$ and $R_{200}$ ), as a function of cluster mass at $z=0$ (left panel) and $z=1$ (right panel). Different colors indicate different simulated physics models: AGN feedback (red), radiative cooling (blue), and shock heating-only (green). Note that $f_{b}$ in our shock heating-only and radiative cooling models are almost identical within the scatter for the integration radii $R_{500}$ and $R_{200}$. In our cooling and star formation model at $z=0$, we find an increasing stellar mass fraction towards smaller clusters that is accompanied by a decreasing gas mass fraction, particularly within $R_{500}$. This trend of a decreasing $f_{\text {gas }}$ towards smaller clusters is amplified in our AGN feedback model, while the stellar mass fraction remains almost constant across our mass range probed. AGN feedback pushes gas out of smaller clusters beyond $R_{200}$ which causes a reduction of the baryon mass fraction in comparison to our model without AGN feedback. In contrast, the trends of $f_{\text {star }}$ with cluster mass are almost absent at $z=1$.

All clusters:

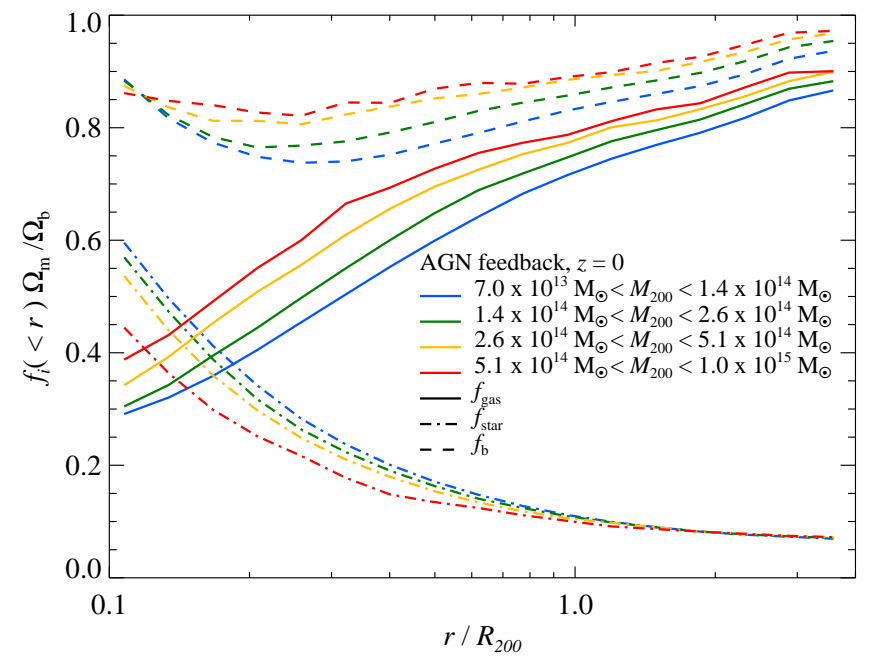

Relaxed clusters:

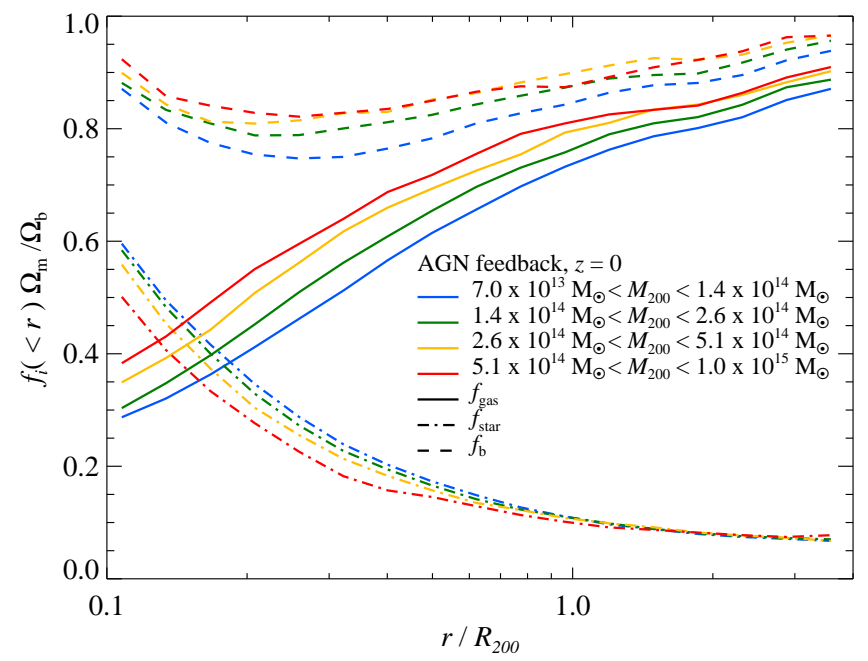

Figure 10. Radial median profiles of the mass fractions in gas $\left(f_{\text {gas }}\right.$, solid $)$, stars $\left(f_{\text {star }}\right.$, dash-dotted $)$, and baryons $\left(f_{b}\right.$, dashed). Each profile is normalized by the universal baryon fraction and different colors correspond to different cluster mass ranges (indicated in the legends). The profiles in the left panel include all clusters and the right panel shows the median of a sub-sample of the third most relaxed clusters, selected by the lowest third in total kinetic-to-thermal energy ratio, $K / U$. In contrast to our non-equilibrium measures such as clumping or kinetic pressure support, the mass fractions in gas, stars, an baryons are almost independent on the clusters' dynamical state.

heating approaches a value of $f_{\text {gas }} / f_{c}=0.93 \pm 0.03(1-\sigma)$ at $r \sim R_{200}$ (which is reduced by one percentage point for larger clusters). These lower values of $f_{\text {gas }}$ represent the pivot point from which our radiative models can reduce $f_{\text {gas }}$ furthermore through conversion of baryons into stars or by expelling it from the cluster region (in the case of our AGN feedback model).

In our radiative cooling model, $f_{\text {gas }}$ increases moderately with cluster mass at $R_{500}$ to level off at a value of $f_{\text {gas }} / f_{c}=0.79$ for big clusters. This trend is weakened at $R_{200}$. The opposite behavior is seen for $f_{\text {star }}$ so that the resulting baryon frac- tion almost exactly sums to a value that is seen in our shock heating-only model. At $R_{200}$, the scatter in $f_{\text {gas }}$ drops by $1-2$ percentage points in comparison to the scatter at $R_{500}$ for both of our radiative models.

While in our AGN feedback model, the effect of a decreasing $f_{\text {gas }}$ towards lower cluster masses is amplified and the trend of an increasing $f_{\text {star }}$ towards lower masses is weakened (at $R_{200}$ ). Figure 10 shows that these trends are still present at smaller radii and become progressively stronger towards the center. Interestingly, these effects partially compensate for the 
baryon fraction, $f_{b}$, which attains a much weaker mass dependence. Comparing the radial profiles of these mass fraction for all clusters (left panel of Figure 10) and a sub-sample of the third most relaxed clusters (right panel) we virtually do not see any differences. In contrast to our non-equilibrium measures such as clumping or kinetic pressure support, the mass fractions in gas, stars, and baryons are almost independent on the clusters' dynamical state. The only exception are the central values for $f_{\text {star }}$ in massive clusters which are higher by $5 \%$ in our relaxed-cluster sample in comparison to all clusters.

However, there are two noticeable differences of the mass fractions in our AGN feedback model in comparison to those in our radiative cooling model (Figure 9). (1) The baryon fractions do not sum up to the value of our shock heating-only model. There is a deficit of gas mass at $R_{200}$ that decreases from $10 \%$ at $M_{200} \simeq 10^{14} \mathrm{M}_{\odot}$ down to $5 \%$ at $7 \times 10^{14} \mathrm{M}_{\odot}$. Only for the largest clusters $\left(M_{200} \simeq 10^{15} \mathrm{M}_{\odot}\right)$ does the baryon fraction of the AGN feedback model correspond to that of the shock heating-only model. As explained in in Section 4.1 this is due to the powerful outflows of gas that deposit considerable amounts of gas beyond $R_{200}$ so that the cosmic mean is not even reached by $4 R_{200}$. The only exception to this are the largest clusters whose gravitational potential provide a strong enough pull on the gas that was successful in having re-accreted the gas. (2) In our AGN feedback model at $R_{200}, f_{\text {star }}$ remains almost independent of cluster mass (in contrast to the values at $\left.R_{500}\right)$.

This behavior is very interesting with respect to its implications for the redshift evolution of $f_{\text {star }}$ on group scales. In order to build up high-mass clusters with low values of $f_{\text {star }} / f_{c} \sim$ $0.1-0.15$, their progenitors also had to have these low stellar mass fractions in a hierarchically growing universe since the amount of baryons locked up in (low-mass) stars remains there throughout a Hubble time in the model of star formation adopted here (Springel \& Hernquist 2003) Since $f_{\text {star }}$ is increasing towards lower cluster masses in our radiative cooling model, this would imply that the groups we observe today, do not reflect the properties of groups at high- $z$ (in terms of $f_{\text {star }}$ ) that merged to assemble large clusters today. This is demonstrated by the almost constant behaviour of $f_{\text {star }}$ as a function of cluster mass at $z=1$ in our radiative cooling model (righthand panel of Figure 9). The values of $f_{\text {star }}$ in groups at $z \simeq 1$ is almost at the same level as $f_{\text {star }}$ for big clusters today. In contrast, the approximate constancy of $f_{\text {star }}$ with cluster mass in our AGN feedback models suggests that groups that are observed today do not need to be different from group-scale progenitor systems of clusters today.

The increasing value of $f_{\text {star }}$ with time on groups scales in our radiative cooling model (Figure 8) also suggests an excess star formation at late-times which results in too blue colors and too massive stellar masses at the high-mass end in comparison to observations (Sijacki et al. 2007). We note that the distributions of $f_{\text {star }}$ and $f_{\text {gas }}$ are almost Gaussian (independent of the models), resulting in almost identical median and mean.

\footnotetext{
2 There can be return of stellar mass to the gaseous phase by means of stellar winds which is thought to mostly sustain star formation in galactic disks (Martig \& Bournaud 2010; Leitner \& Kravtsov 2011). If there is a considerable population of intracluster stars (Puchwein et al. 2010), stellar mass loss could be a potential channel of increasing $f_{\text {gas }}$ at the expense of $f_{\text {star }}$, but the low observed mass fraction of intracluster stars renders it unlikely to be an important contribution, especially on the high-mass cluster end (Lin et al. 2003; Zibetti et al. 2005;; Gonzalez et al. 2005, 2007; Giodini et al. 2009).
}

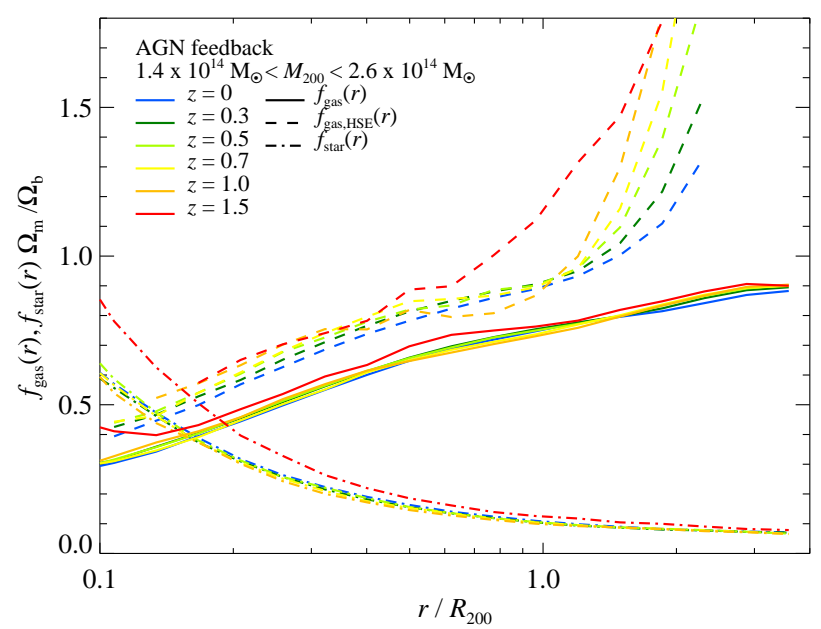

Figure 11. In our AGN feedback model, there is little redshift evolution in the median profiles of the gas mass fraction, $f_{\text {gas }}(<r)$, (solid) and stellar mass fraction, $f_{\text {star }}(<r)$ (dash-dotted), when radii are scaled to $R_{200}$. Both quantities are normalized by the universal baryon fraction and shown as a function of redshift for fixed mass range (different colors). In contrast, the profiles $f_{\text {gas }}(<r)$ that assume hydrostatic equilibrium (dashed) show a larger bias at high redshift due to the greater kinetic pressure contribution at earlier times. Those probe on average dynamically younger objects that are in the process of assembling.

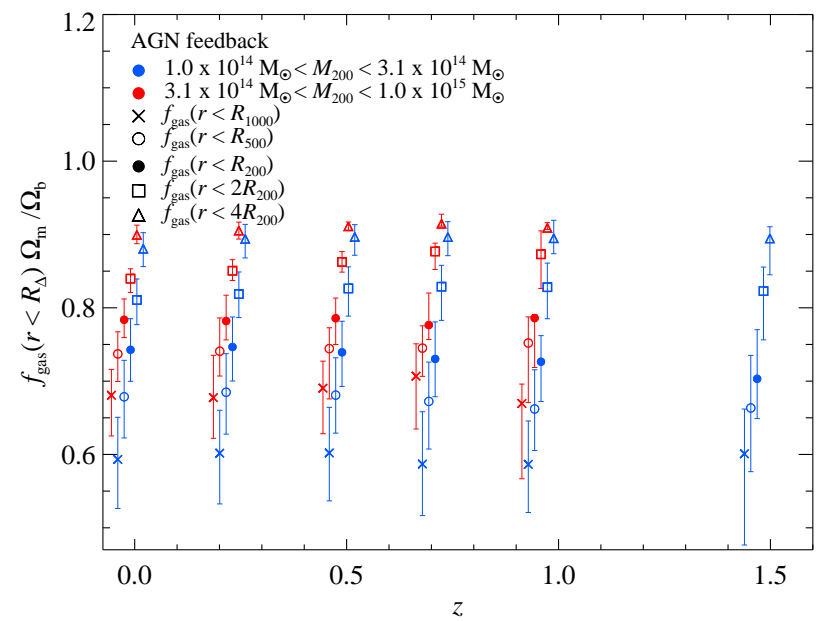

Figure 12. Redshift evolution of the gas mass fractions, $f_{\text {gas }}(<r)$, that are computed within different radii $\left(R_{1000}, R_{500}, R_{200}, 2 R_{200}\right.$, and $\left.4 R_{200}\right)$ for our AGN feedback model. We contrast median values for groups $\left(1 \times 10^{14} \mathrm{M}_{\odot}<\right.$ $M_{200}<3.1 \times 10^{14} \mathrm{M}_{\odot}$, blue) and clusters $\left(3.1 \times 10^{14} \mathrm{M}_{\odot}<M_{200}<1 \times\right.$ $10^{15} \mathrm{M}_{\odot}$, blue) and find very little redshift evolution for $f_{\text {gas }}(<r)$, particularly for large clusters. While $f_{\text {gas }}$ increases with increasing radius, the baryon mass fraction does not reach the cosmic mean for $r<4 R_{200}$ since the stellar mass fraction is $f_{\text {star }}\left(<4 R_{200}\right) \simeq 7 \%$.

\subsection{Redshift evolution of gas and stellar mass fractions}

First, we show the redshift evolution of the median profiles of the stellar and gas mass fractions in our AGN feedback model at fixed cluster mass, $1.4 \times 10^{14} \mathrm{M}_{\odot}<M_{200}<2.6 \times 10^{14} \mathrm{M}_{\odot}$ (Figure 11). To address the cluster-to-cluster scatter, we additionally show the redshift evolution of the median and percentiles containing $68.3 \%$ probability centered on the median (corresponding to 1- $\sigma$ intervals) for small and big clusters separated at the characteristic mass of $M_{200}=3.1 \times 10^{14} \mathrm{M}_{\odot}$ (Figure 12). 
For a given cluster-mass bin and with increasing redshift, we are probing clusters with a higher mass accretion rate on average, i.e., dynamically younger systems. Back to $z=1$, there is almost no evolution in these mass fractions, especially for bigger clusters and larger radii. The constancy of $f_{\text {star }}$ implies that the integral of the galaxy mass function increases at the same rate as the DM mass, which is realized if most of the stellar mass is already in place by the time it assembles in the cluster halo (since we do not account for stellar mass loss in our simulations).

How does this finding compare to observations? Optical observations find an abundance of red elliptical galaxies within clusters that is the foundation for the red sequence cluster finding algorithm (Gladders \& Yee 2000). This is the standard cluster finding method in the optical for $z \leq 1$ and has been extended to higher redshifts by the Spitzer Adaptation of the Red-sequence Cluster Survey (SpARCS, Muzzin et al. 2009) and photometric cluster finding techniques such as the IRAC Shallow and Deep Cluster Surveys (ISCS and IDCS, Brodwin et al. 2006; Mancone et al. 2012). In the literature there is a controversy about the faint-end slope of the red sequence, which translates into an uncertainty of the builtup of stellar mass in observations across cosmic time. Some groups find a constant faint-end slope of the red sequence (e.g., Stanford et al. 1998; Andreon 2006, 2008; De Propris et al. 2013) after accounting for surface brightness selection on red sequence luminosity function while others observe a decrease of the faint end of the red sequence (De Lucia et al. 2004, 2007; Tanaka et al. 2004; Stott et al. 2007; Gilbank et al. 2008; Rudnick et al. 2009; Papovich et al. 2010; Vulcani et al. 2011; Rudnick et al. 2012). Those authors infer an evolving faint end of the red sequence, which they interpret as a built-up of stellar mass in the red sequence with a stellar mass growth by factor of two between $z=1.6$ down to 0.6 and another factor of two since then. This agrees on average with the mass assembly history of cluster halos after accounting for stellar mass loss.

In our simplified star formation models, we do not account for stellar mass loss, which can return up to $30 \%-50 \%$ to the ambient medium, depending on the initial mass function. We emphasize that even if our stellar mass prescription and hence $f_{\text {star }}$ may be off by $50 \%$ due to the effect of stellar mass loss or limiting resolution, this would bias the gas mass fractions by a factor of at most $\Delta f_{\text {star }} f_{\text {star }} / f_{\text {gas }} \lesssim 0.2$. However, this factor will be substantially reduced in practice because a larger (smaller) amount of formed stellar mass is partly balanced by an increased (decreased) inflow of gas from larger radii to maintain the approximate pressure balance due to the increased gas mass loss to stars. This renders our results with respect to $f_{\text {gas }}$ quite robust.

We demonstrate in Figure 12 that there is weak evolution of $f_{\text {gas }}$ (at the level of $1-\sigma$ ) for our small clusters at $R_{200}$ which disappears for larger radii or larger clusters. In particular for clusters with $M_{200}>3.1 \times 10^{14} \mathrm{M}_{\odot}$, we see no evolution of the median $f_{\text {gas }}$ already for radii as small as $R_{500}$. At $z=1.5$, both $f_{\text {star }}$ and $f_{\text {gas }}$ are increased towards the center indicating that those redshifts probe the tail end of the epoch of our AGN feedback which is most efficient at $z \geq 2$ in blowing gas out (Figure 11, see also McCarthy et al.2011). At $z>1.5$, we run out of statistics for the mass range under consideration. However, this interpretation is confirmed by looking at a smaller cluster mass range at an even higher redshift which shows a further increase in the stellar mass fraction.

This finding is also consistent with the amount of energy in- jected by AGN feedback over the simulation time. Approximately one third of total energy is delivered in the cluster formation phases at $z>2$ (analogous to high- $z$ quasar-like feedback), another third in the redshift range $1<z<2$, and the final third below $z=1$ (analogous to jet/bubble like feedback). These fractions depend moderately on the numerical resolution; increasing the resolution enables to resolve the growth of smaller halos at earlier times and causes a higher fraction of energy injection at higher redshifts (see Battaglia et al. 2010, for a discussion).

Despite the almost constant evolution of the gas fractions for $r \gtrsim R_{200}$, there is positive evolution of the biased gas mass fraction $f_{\text {gas,HSE }}$ at these radii due to the evolution of the kinetic-tothermal pressure ratio when radii are scaled to $R_{200}$ (Figure 11). Most of this apparent evolution can be absorbed by redefining $R_{200}$ as the radius at which the mean interior density equals $\Delta$ times the mean density of the universe (see Figure 21 in BBPS1). However, this rescaling would then imply a redshift evolution of $f_{\text {gas }}$ with smaller values of $f_{\text {gas }}$ at higher redshifts.

\subsection{Comparison to data}

In Figure 13, we compare our AGN feedback model with data. We compare radial $f_{\text {gas }}$ profiles of clusters that are sorted in two bins according to their spectroscopic temperatures, $2.5<k T_{\text {spec }} / \mathrm{keV}<5.0$ and $k T_{\text {spec }}>5.0 \mathrm{keV}$. We use the $T_{\text {spec }}-M_{500}$ relation by Vikhlinin et al. (2009a) to translate our $M_{500}$ values to spectroscopic temperatures. We adopt a $15 \%$ correction to the X-ray-inferred $M_{\mathrm{HSE}}$ estimates Kravtsov et al. 2006), which is valid for the respective observational sample selection criterion. Thus, the spectroscopic temperatures $2.5 \mathrm{keV}$ and $5 \mathrm{keV}$ correspond to $\sim 1.7 \times 10^{14} \mathrm{M}_{\odot}$ and $\sim 5.0 \times 10^{14} \mathrm{M}_{\odot}$, respectively.

The left panels of Figure 13 show gas mass fractions from individual X-ray cluster observations that combine surface brightness and temperature maps of deep Chandra Vikhlinin et al. 2006; Allen et al. 2008) and XMM-Newton data of a representative sample of nearby X-ray galaxy clusters (REXCESS, Pratt et al. 2010) 3 There is generally good agreement of our model clusters populating the high-temperature bin, especially with the Chandra data. In contrast, our simulated small-mass clusters tend to have on average slightly too high $f_{\text {gas }}$ values. However, these Chandra data represent a biased sample since Vikhlinin et al. (2006) and Allen et al. (2008) targeted extremely relaxed cool core clusters for their mass measurements. REXCESS, on the other hand, should be unbiased for X-ray selected systems. Indeed, our model $f_{\text {gas }}$ profiles compare similarly well to the REXCESS cool core systems while we overproduce the apparent $f_{\text {gas }}$ estimates in morphologically disturbed systems (independent of cluster mass respectively spectroscopic temperature). It is unclear whether the cause for the discrepancy is on the data or simulation side. There is the possibility that our simulations fail to reproduce the non-cool core systems while they apparently match the $f_{\text {gas }}$ characteristics of cool core systems. However, statistically, we find no evidence that the true $f_{\text {gas }}$ is considerably changed as a result of the cluster's dynamical state (Figure 1). On the other side, it remains to be seen whether spherical profiles of these

\footnotetext{
${ }^{3}$ We separate the XMM-Newton clusters additionally into cool core and morphologically disturbed systems. The cool core definition is based on scaled central density, and the morphologically disturbed definition is based on centroid shift, $\langle w\rangle$, following Pratt et al. (2009). The two clusters that are classified as both cool core and morphologically disturbed systems, are presented as being morphologically disturbed.
} 
Data comparison of radial $f_{\text {gas }}$ profiles:
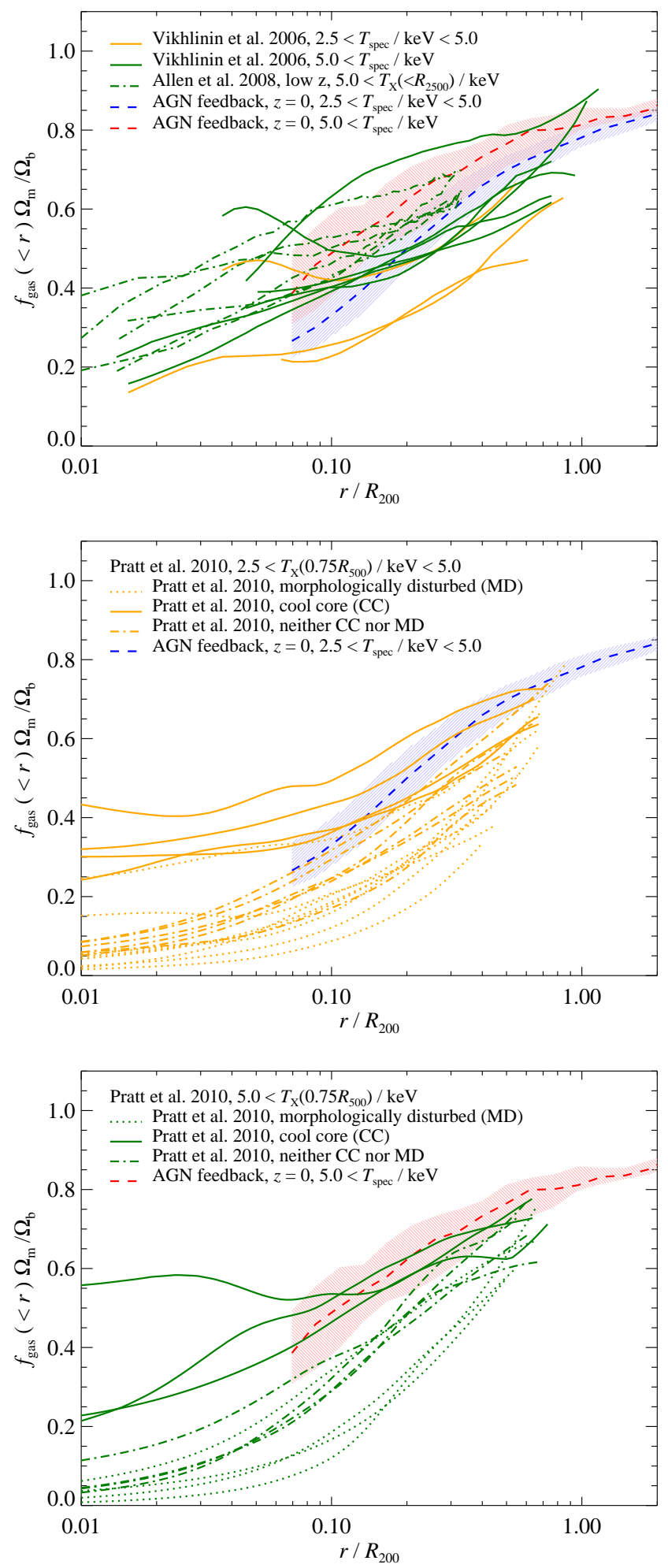

Data comparison of enclosed mass fractions:
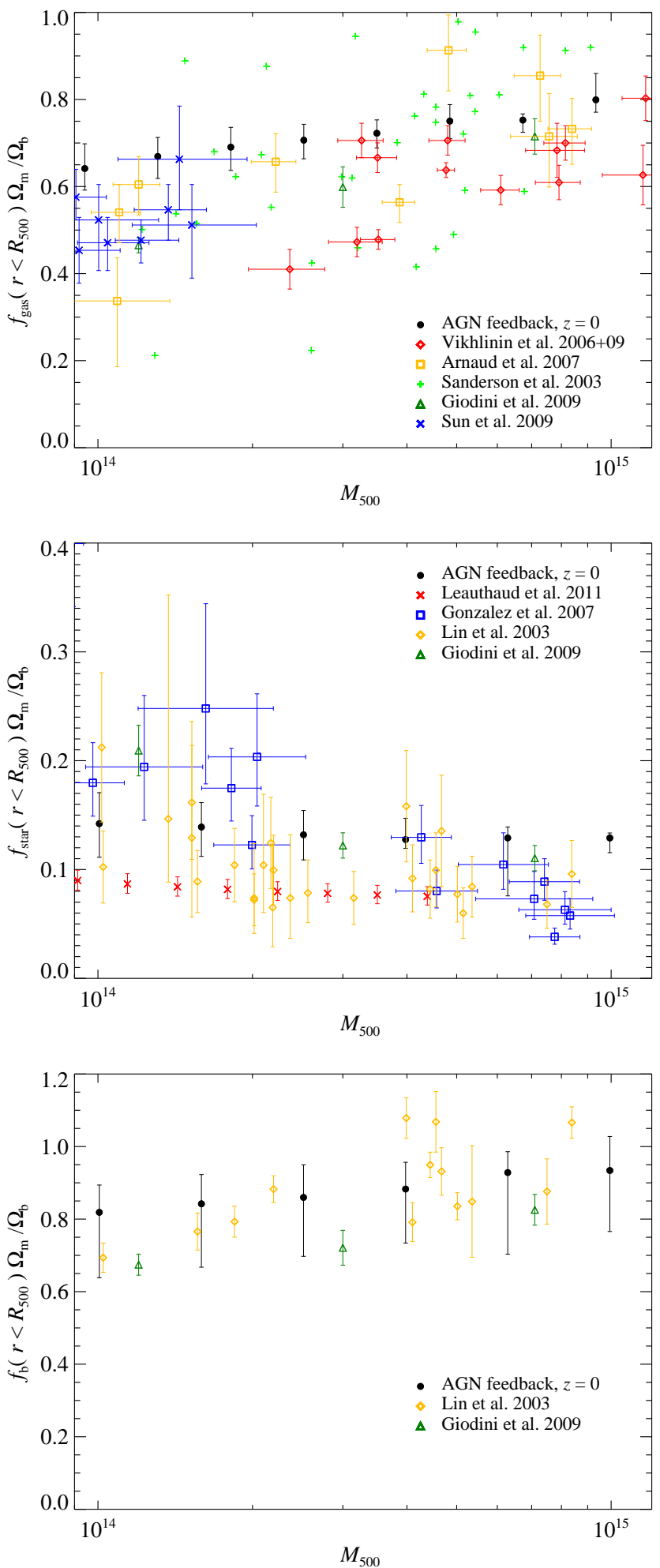

Figure 13. Comparison of our AGN feedback model with data. In the left panels, we compare radial $f_{\text {gas }}$ profiles of two cluster temperature bins that are separated by spectroscopic temperature $T_{\text {spec }}=5 \mathrm{keV}$ (using the $T_{\mathrm{spec}}-M_{500}$ relation by Vikhlinin et al. (2009a) for translating our $M_{500}$ values). The top panel shows Chandra data from Vikhlinin et al. (2006) and Allen et al. (2008). The middle and bottom panels shows the XMM-Newton data of the REXCESS cluster sample (Pratt et al. 2010), separated by spectroscopic temperature $T_{\mathrm{spec}}=5 \mathrm{keV}$, and differentiated by dynamical state. Right panels: we compare the cluster mass dependence of enclosed gas mass fractions (top), stellar mass fractions (middle), and consistent treatments of the baryon mass fractions, i.e., $f_{b}$ obtained from the same objects, respectively (bottom). We use $f_{\text {gas }}$ data by Sanderson et al. (2003); Vikhlinin et al. (2006, 2009a); Arnaud et al. (2007); Giodini et al. (2009); Sun et al. (2009) and $f_{\text {star }}$ data by Lin et al. (2003); Gonzalez et al. (2007); Giodini et al. (2009); Leauthaud et al. (2011). Overall, our AGN feedback model successfully reproduces observed values and trends, albeit with slightly higher values of $f_{\text {gas }}$ at the mass scales of groups. While the scatter of our simulated distribution is noticeably smaller, we caution that we did not include measurement biases that would substantially add to the simulated scatter. 
inherently non-spherical merging systems bias the derived values for $f_{\text {gas }}$ low.

In Figure 14, we compare our models with $f_{\text {gas }}$ estimates from the combination of Planck Sunyaev-Zel'dovich data and different X-ray observations of massive clusters $\left(k T_{\text {spec }} \gtrsim\right.$ $5 \mathrm{keV})$. Depending on the availability of X-ray spectra, different approaches have been pursued in the literature. The Planck collaboration used XMM-Newton data interior to $R_{500}$ with spectroscopic temperature profiles (Planck Collaboration et al. $2013 \mathrm{~b}$ ) and extrapolated these beyond $R_{500}$ while assuming two hypotheses that are likely bounding the true profile 4 In combination with deprojected Planck pressure profiles, this yields gas density profiles and-through integration- $-M_{\text {gas }}(<r)$. The total mass profile is assumed to follow a generic NFW model (Navarro et al. 1997), which is normalised to $M_{500}$ from the $M_{500}-Y_{X}$ scaling relation (Arnaud et al. 2007, 2010). The resulting $f_{\text {gas }}$ profile is somewhat lower than that of our AGN simulations for $r<R_{500}$ while it agrees with the simulation model at larger radii. Since our simulated pressure profiles match the observed ones (Planck Collaboration et al. 2013b), the reason for the discrepancy could either lie in the assumptions used to derive $f_{\text {gas }}$ or that our simulations yield too high values for $f_{\text {gas }}$. Possibilities for observational biases include incomplete accounting for multi-temperature phases in cluster outskirts and biases of the total mass profile. $M_{\text {tot }}$ could be biased by neglecting the substantial scatter among individual DM density profiles as well as not accounting for the covariances between the different parameters adopted for the DM profile such as mass-concentration and $M_{500}-Y_{X}$ scaling relation.

Using ROSAT data (that lacks spectroscopic X-ray information) of a sample of 18 clusters that also has Planck SunyaevZel'dovich data, Eckert et al. (2013) follow a different methodology. This uses the hydrostatic equilibrium mass,

$$
M_{\mathrm{HSE}}(<r)=-\frac{r^{2}}{G \rho_{\text {clump }}} \frac{d P_{\mathrm{th}}}{d r},
$$

where $\rho_{\text {clump }}$ derives from the X-ray emission measure and is subject to residual density clumping (that escaped the masking in the X-ray analysis). The thermal pressure gradient is obtained from the Planck best-fit generalized NFW models for the pressure, which does not depend on clumping. The gas mass fraction is derived via $f_{\text {gas }}(<r)=M_{\text {gas,clump }}(<r) / M_{\mathrm{HSE}}(<r)$, where $M_{\text {gas,clump }}(<r)$ is given by Equation (9). Hence, the gas mass fraction obtained by this method scales approximately with the (residual) clumping factor,

$$
f_{\text {gas }}(<r) \propto \sqrt{C_{2, \rho}(r)} \int_{0}^{r} \sqrt{C_{2, \rho}\left(r^{\prime}\right)} \rho_{\text {true }}\left(r^{\prime}\right) r^{\prime 2} d r^{\prime},
$$

rather than the square root as in the case of pure X-ray based analysis (where the pseudo pressure scales also with the residual $\sqrt{C_{2, \rho}}$, canceling the clumping factor dependence in $\left.M_{\mathrm{HSE}}\right)$. Additionally, $f_{\text {gas }}$ is biased high owing to the hydrostatic mass bias, i.e. by neglecting the kinetic pressure support (see Figure 1 or Rasia et al. (2006); Vazza et al. (2011a); Nelson et al. (2012)) and the non-thermal pressure contributed by a potential relativistic particle population in cluster outskirts (Pfrommer et al. 2007; Pinzke \& Pfrommer 2010; Vazza et al. 2012). As before for the individual Chandra and XMM-Newton clusters, our simulation models provide a good match to the

4 Note that we use an updated version of $f_{\text {gas }}(<r)$ as presented by the Planck Collaboration et al. (2013c). median of six cool core systems by Eckert et al. (2013). However, their median profile of twelve non-cool core clusters shows larger values than predicted by our simulations for radii $\gtrsim 0.7 R_{200}$. This is especially remarkable since there is a substantial discrepancy between the non-cool core systems in the sample by Eckert et al. (2013) and the morphologically disturbed systems of Pratt et al. (2010), some of which may be explained by the different dependencies on the clumping factor as discussed here. We caution that more careful X-ray mocks (exactly quantifying the various biases discussed here) are needed to draw final conclusions regarding the direct comparison of our simulated models with observations.

In the right-hand panels of Figure 13, we compare the cluster mass dependence of enclosed gas mass fractions (top), stellar mass fractions (middle), and baryon mass fractions (bottom). Note that we only use those compilations of $f_{b}$ versus mass where the gas and stellar masses have been obtained from the same clusters, respectively. Overall, our AGN feedback model successfully reproduces observed values and trends, albeit with slightly higher values of $f_{\text {gas }}$ (and to a lesser extent $f_{b}$ ) at the mass scales of groups in comparison to X-ray observations (cf. Arnaud et al. 2007; Sun et al. 2009). While the scatter of our simulated distribution is noticeably smaller, we caution that we did not include measurement biases that would substantially add to the simulated scatter as demonstrated in Section 3 .

There could be several explanations for an overshooting of our simulated $f_{\text {gas }}$ values at group scales. Those could include numerical resolution, shortcomings of the AGN modeling, or missing physics in the simulations. Increasing resolution would enable us to resolve the formation of smaller objects earlier on which may allow a more vigorous blow-out of the gas already in halos in the mass range $10^{12} \lesssim M_{200} / \mathrm{M}_{\odot} \lesssim$ $10^{13}$. Those are just above the knee of the galaxy luminosity function and hence have a decreasing efficiency of star formation per unit halos mass in more massive halos which is usually attributed to AGN feedback (e.g., Bower et al. 2012; Puchwein \& Springel 2013).

As laid out in Section 2, we use an extremely low-resolution incarnation of AGN feedback that enables us to simulate large volumes and cluster samples, which are needed to provide precise predictions for the next generation X-ray and SZ surveys. Our AGN feedback prescription couples the thermal energy feedback to the global SFR of the central cluster region. While this model is able to arrest overcooling in massive clusters, it may be too simplified for groups. The observed (low-frequency) radio emission that spatially coincides with Xray cavities suggests the presence of non-thermal components composed of cosmic rays and magnetic fields in these lobes. Modelling AGN feedback into cosmic ray rather than thermal energy (Sijacki et al. 2008) or exploring kinetic jet feedback that thermalizes at larger radii (Dubois et al. 2010) may be viable alternatives to our simulated scenario, but would also require better spatial resolution. If cosmic rays are injected into the ICM by the break-up of radio bubbles due to KelvinHelmholtz instabilities, they can stream at the Alfvén velocity with respect to the plasma rest frame and heat the surrounding thermal plasma at a rate that can balance that of radiative cooling on average (as demonstrated for the Virgo cluster), thereby providing a physical solution to the "cluster cooling flow problem" (Loewenstein et al. 1991; Guo \& Oh 2008; Pfrommer 2013).

Finally, our model may be missing important physics that could additionally add entropy to the centers of groups, such 

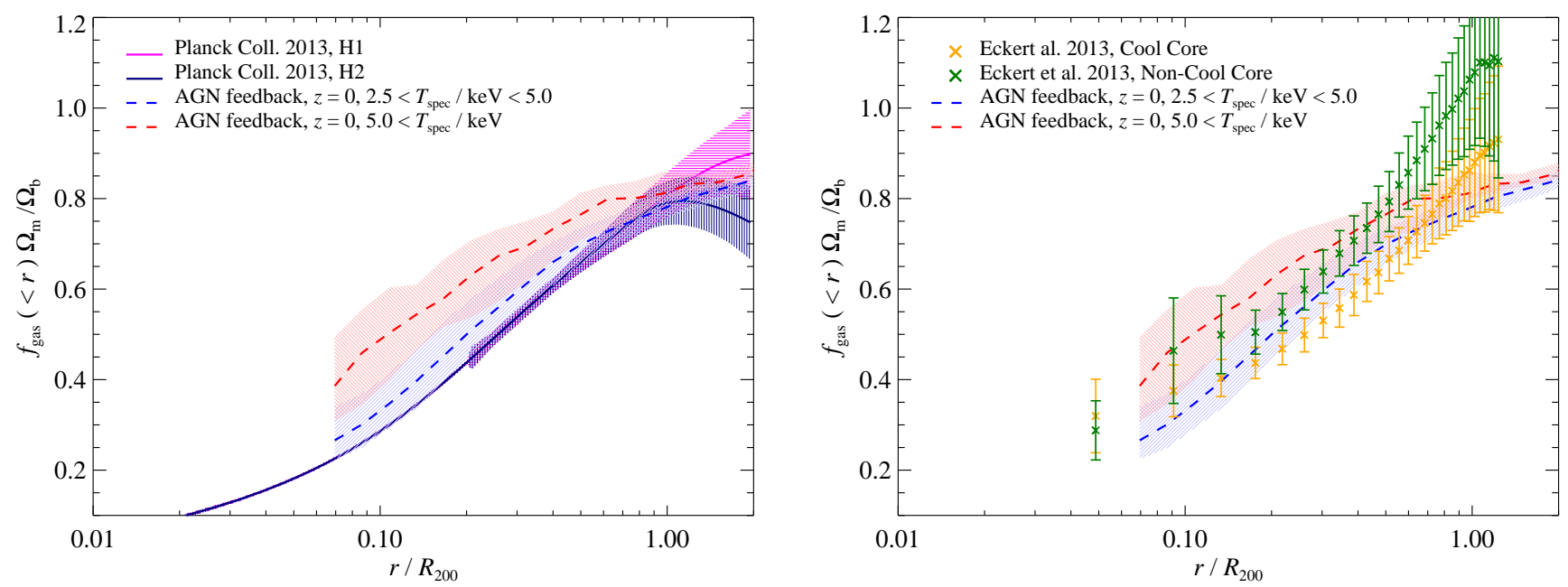

Figure 14. Comparison of radial $f_{\text {gas }}$ profiles in our AGN feedback model and data that combine different X-ray and Planck Sunyaev-Zel'dovich observations of massive clusters $\left(k T_{\text {spec }} \gtrsim 5 \mathrm{keV}\right)$. Left panel: the gas density profile is obtained by combining the pressure profile derived from Planck data with spectroscopic temperature profiles interior to $R_{500}$ from XMM-Newton data (Planck Collaboration et al. 2013b). Two differing hypotheses (H1 and H2) have been adopted to extrapolate those beyond $R_{500}$, bounding the plausible range. $\mathrm{H} 1$ extrapolates the average best fit model across the sample from X-ray spectroscopy and $\mathrm{H} 2$ assumes a constant temperature beyond $R_{500}$ that is equal to the average temperature measured in the last radial bin across the sample. Enclosed gas fractions are derived by dividing $M_{\text {gas }}(<r)$ with the total enclosed mass, assuming a generalized NFW model and a $M_{500}-Y_{X}$ scaling relation. Right panel: $f_{\text {gas }}$ is derived by combining Planck-inferred pressure profiles and ROSAT X-ray data (Eckert et al. 2013) while assuming hydrostatic equilibrium and neglecting the density clumping bias.

as the recently suggested (Broderick et al. 2012; Chang et al. 2012; Pfrommer et al. 2012; Puchwein et al. 2012) blazar heating model that proposes the bolometric luminosity of $\mathrm{TeV}$ blazars as an additional heating source of the inter-galactic medium. Phenomenologically, highly biased regions that first turn-around to form groups and clusters should be heated first. This would imply an evolving pre-heated entropy floor and cause an additional decrease of $f_{\text {gas }}$ in late-forming groups that are observed on a sufficiently short time scale after formation so that the central group medium had not have time to enter the strongly cooling regime (Pfrommer et al. 2012).

\subsection{Comparison to previous work}

Given the importance of cluster X-ray observations for cosmological parameter estimation, there has been a comparably large body of work concerning gas fractions in cosmological cluster simulations. Early work on $f_{b}$ in non-radiative simulations showed a strong systematic discrepancy between different numerical methods. This is surprising since all codes should solve the same equations for the gas and collisionless DM physics without any ambiguity in formulating a subgrid description of star formation and associated feedback processes. SPH simulations of ten massive clusters in a $\Lambda$ CDM cosmology derived values of $f_{b} \approx 0.87$ and 0.83 within $R_{\text {vir }}$ and $0.5 R_{\mathrm{vir}} \approx R_{500}$, respectively, and show only a very weak evolution of $f_{b}$ from $z=1$ to 0 (Eke et al. 1998). A number of further studies that all employ the energy formulation of SPH confirm these findings (Frenk et al. 1999; Bialek et al. 2001; Muanwong et al. 2002). In contrast, cluster studies that employ the adaptive mesh refinement (AMR) technique for solving the hydrodynamic equations find higher values of $f_{b} \approx$ $0.97 \pm 0.03$ and $0.94 \pm 0.03$ within $R_{\text {vir }}$ and $0.5 R_{\text {vir }} \approx R_{500}$, respectively (Kravtsov et al. 2005). Cluster simulations with the entropy-conserving SPH code GADGET yield values of $f_{b} \approx 0.92-0.93$, higher by about 5 percentage points than the energy-conserving formulation of SPH but still smaller than the AMR results (see Kay et al. 2004; Kravtsov et al. 2005, which is also in line with our findings). All the different methods find no significant redshift evolution of the baryon fraction in non- radiative simulations.

Simulations with radiative cooling and star formation necessarily yield larger discrepancies in the resulting $f_{b}$ values owing to the larger uncertainties of implementing subgrid star formation and feedback processes. Common to all of these approaches is that too many stars condense out of the ICM, which results in the "overcooling problem", i.e., $f_{\text {gas }}$ values that are too small and $f_{\text {star }}$ values that are too high in comparison to observations. SPH simulations (employing the energy formulation) with radiative cooling or feedback yield values of $f_{b} \approx 0.85-0.9$ for the cluster mass range considered here (see Figure 3 of Muanwong et al.2002). For smaller galaxy clusters (within a factor of two of mass $M_{200}=10^{14} h^{-1} \mathrm{M}_{\odot}$ ), entropyconserving SPH simulations by Kav et al. (2004) and AMR simulations by Kravtsov et al. (2005) find similar average radial profiles of $f_{\text {gas }}$. However, those results differ for larger clusters and the AMR results obtain substantially higher $f_{\text {gas }}$ values. Ettori et al. (2006) presented an analysis for a sample of galaxy clusters simulated with the entropy-conserving GADGET SPH code that followed radiative cooling and star formation (essentially similar to our radiative cooling model). They find total baryon fractions of $f_{b}\left(<R_{\mathrm{vir}}\right) \approx 0.93-0.95$, similar to our values and those by Kravtsov et al. (2005) on cluster-mass scales. The gas fractions found in Ettori et al. (2006) and more recently in Sembolini et al. (2013) of 0.75-0.8 agree with our values (for the radiative cooling model), but are higher than the corresponding values by Kravtsov et al. (2005) because of the different implementations of cooling and star formation.

More recently, baryon fractions have been studied in AGN feedback models. Puchwein et al. (2008) presented mock Xray observations of a sample of zoomed cluster simulations which account for radiatively cooling gas of primordial composition, star formation, and thermal energy feedback by black holes that grow through Bondi-Hoyle accretion. These simulations produce gas mass fractions that match the observations and are also in agreement with our AGN feedback results for larger clusters $\left(M_{500} \gtrsim 2 \times 10^{14} \mathrm{M}_{\odot}\right)$. However, our smaller systems appear to have slightly larger values of $f_{\text {gas }}$ due to the coarser resolution of our cosmological simulations in compar- 
ison to those zoomed simulations that are able to resolve AGN feedback at earlier times in galaxies above $L^{*}$. In contrast, our stellar mass fractions on cluster scales match the observed values, but are smaller by about a factor of three in comparison to the AGN feedback simulation by Puchwein et al. (2010) (see their Figure 5). Improving the simulated physics of these AGN feedback models, Planelles et al. (2013) follow the detailed chemical evolution of the IGM and self-consistently account for metallicity-dependent radiative cooling (thus improving the simplifying assumptions of collisional ionization equilibrium and solar relative abundances, which were underlying earlier work by Fabjan et al. 2010). On cluster-mass scales, they find slightly smaller gas fractions than ours and those by Puchwein et al. (2008). Additionally, they have similarly large stellar mass fractions as Puchwein et al. (2010), which appear to be in conflict with the data. Common to all that body of work (including ours) is that it estimates the black hole accretion rate and hence the amount of feedback energy directly from simulated hydrodynamical quantities (i.e., entropy or density) by means of a subgrid accretion model, which ensures a substantial predictive power of this approach. In contrast, Young et al. (2011) derive their feedback energy from a semi-analytical model of galaxy formation that they couple to hydrodynamical simulations. By construction, their approach suffers less from resolution effects in comparison to ours and produces a strong cluster-mass dependence of $f_{\text {gas }}\left(<R_{\Delta}\right)$ for various characteristic cluster radii, in agreement with the observations, particularly on group scales. However, their models appear to have problems in reproducing the larger $f_{\text {gas }}$ values of some of the observed $f_{\text {gas }}$ profiles in large clusters with $T_{\text {spec }}>5 \mathrm{keV}$ (Vikhlinin et al. 2006; Allen et al. 2008).

Summarizing, it appears that there are still systematic differences between Lagrangian SPH and Eulerian AMR codes in computing baryon fraction in non-radiative simulations. Those however are reduced to the level of about five percentage points when AMR results are compared to those obtained with the entropy-conserving GADGET SPH code. Since this is the baseline model out of which radiative cooling starts to condense cool gas and to form stars, more work is needed to fully understand the reason of the discrepancy. Purely radiative models, which only account for star-formation feedback, suffer from the over-cooling problem and produce stellar and gas mass fractions in conflict with observations. Instead, a growing body of AGN feedback models demonstrates that the overcooling problem can be weakened or even circumvented, depending on the detailed implementation of cooling and feedback. Moreover, since that type of physics causes larger changes in $f_{b}$ and $f_{\text {gas }}$ in comparison to the systematic differences owing to the numerical method, this holds the promise that the simulations are starting to produce robust and predictive results (for a certain implementation of AGN feedback) so that a comparison to observations is a meaningful and well-posed task.

\section{CONCLUSIONS}

In this paper, we performed a study of gas and stellar mass fractions in clusters with the goal to address (1) the bias of $f_{\text {gas }}$ measurements in recent Suzaku X-ray measurements of the ICM beyond the virial radius and (2) provide a theoretical framework for using gas masses as a tightly correlated and unbiased proxy for the halo masses. This property lies at the heart of using X-ray-inferred gas masses to derive precision cosmological parameters and is of critical importance for SZ surveys as $f_{\text {gas }}$ sets the normalization of the $Y-M$ scaling relation and the $\mathrm{SZ}$ power spectrum scales with the square of $f_{\text {gas }}$.

\subsection{Biases in $X$-ray inferred gas mass fractions}

We identified two main sources of bias for X-ray inferred values of $f_{\text {gas }}$ in addition to sample variance of clusters and angular orientation variance within clusters; all of which can be addressed with cosmological simulations of a large statistical sample of clusters. (1) Adopting hydrostatic equilibrium masses, i.e., neglecting the non-thermal pressure, biases the inferred masses low by $20-25 \%$ on average (or $15-20 \%$ if we considered only a sample of the third most relaxed clusters). Hence $f_{\text {gas }}$ is biased high by the same factor that increases dramatically for even larger radii due to the larger relative contribution of kinetic pressure. (2) The presence of gas density clumping biases the X-ray surface brightness and hence the inferred density and gas masses high. Hence, the resulting values for $f_{\text {gas }}$ are also biased high at a level of $\sim 10-20 \%$ within $R_{200}$ (slightly mass-dependent and at the low-end for relaxed clusters). For the most massive clusters with $M_{200} \gtrsim 3 \times 10^{14} \mathrm{M}_{\odot}$, it increases even more steeply outside $R_{200}$ in comparison with the bias by HSE masses.

While these two processes represent an upward measurement bias of $f_{\text {gas }}$ in comparison to its cosmic value at $R_{200}$, we also consider the sample variance of the true $f_{\text {gas }}$ as well as its biased measurement across clusters and within clusters for different angular directions. Interestingly, the cluster-to-cluster scatter of the true $f_{\text {gas }}$ decreases for larger radii to values around 5\% at $R_{200}$. In contrast, the cluster sample variance of the biased measurement of $f_{\text {gas }}$ due to the assumption of HSE masses and neglecting density clumping remains fairly constant as a function of radius at a level of $\sigma_{f_{\text {gas }}} / f_{\text {gas }} \simeq 0.1-0.2$ (depending on the dynamical state and mass of clusters) within $R_{200}$ and even starts to increase for larger radii due to the strong clumping term.

These different measurement biases are all manifestations of two underlying physical processes which both lead to significant angular variance of the (gas and total) mass distributions. First, there is a strong internal baryon-to-dark-matter density bias, a consequence of collisional-to-collisionless physics as a result of the differing efficiencies of gas thermalization and the relaxation processes of the DM component. Second, hierarchical structure formation implies that cluster are the latest object that had have time to form until now which manifests itself in a considerable anisotropy of the mass distributions, in particular around the virial radius where the filamentary cosmic web connects to the cluster interiors. The accretion shock of gas that accretes along filaments forms further inwards, leaving a larger level of clumping and kinetic pressure contribution already around $R_{200}$. Simultaneously, this causes a considerable measure of ellipticity (as measured by the eigenvalues of the moment-of-inertia tensor) throughout the clusters (BBPS1).

Equivalently, computing the mass profiles in 48 cones, whose footprints partition the sphere, we find anisotropic gas and total mass distributions that imply an angular variance of $f_{\text {gas }}$ at the level of $30 \%$ (almost independent of radius and cluster mass). Since the angular variance as well as the outliers of the gas and DM mass distribution in angular cones is larger than the angular variance of $f_{\text {gas }}$, this implies spatial correlations of the DM and gas distribution through self-bound substructures that are still holding on to (some) of their gas, especially outside $R_{200}$. We also demonstrated that in the most extreme cases, the cone profiles of $f_{\text {gas }}$ can be biased high by a factor of two in massive clusters $\left(M_{200} \sim 10^{15} \mathrm{M}_{\odot}\right)$. This factor 
is lowered by considering projection effects. The $3 \mathrm{D}$ angular variance drops from $30-35 \%$ at $R_{200}$ down to $\sim 15-20 \%$ in case of the projected (2D) variance. However, hydrostatic mass bias as well as density clumping may (partially) compensate for these projection effects. If the anisotropic mass distribution is indeed responsible for some of the high $f_{\text {gas }}$ values inferred from Suzaku measurements along individual radial arms, it predicts a significantly lower level of $f_{\text {gas }}$ along other directions. This prediction can be tested by increasing the number of radial arms in a given cluster.

We caution that the magnitude of the angular variance of the mass distributions is not independent of the cluster variance, and hence is not additive. Instead, both variances are complementary ways of characterizing the non-equilibrium processes in clusters (anisotropy, kinetic pressure contribution, clumping) that become increasingly complicated at the virial radius and beyond (BBPS4). We also emphasize that the distributions of mass profiles in angular cones is positively skewed (there are more extreme positive outliers in $M_{\mathrm{gas}}$ and $M_{\text {tot }}$ cone profiles) which is expected from the presence of substructures. In particular, the outliers with high values of the mass profiles in individual cones likely correspond to filaments that channel the accreting substructures to the cluster (BBPS4).

Alternatively the increased level of X-ray surface brightness towards the virial region may be due to instrumental or observational effects, such as of unresolved X-ray point sources, point-spread function leakage from masked point sources, or stray light from the bright inner cluster core.

\subsection{Gas and stellar mass fractions in cosmological simulations}

We find little redshift dependence of the radial profile of $f_{\text {gas }}$ in our AGN feedback model, if radii are scaled to $R_{200}$. In particular, this is the case for big clusters with $M_{200} \gtrsim 3 \times 10^{14} \mathrm{M}_{\odot}$ or for larger containment radii $r \gtrsim R_{500}$, or for both. The redshift independence of $f_{\text {gas }}$ depends critically on the ability to scale the radial variable with $R_{200}$ as the $f_{\text {gas }}$ profile increases steadily with radius. If the redshift-dependent systematics of inferring $R_{200}$ can be controlled, this provides a good theoretical basis of using the gas mass fraction to do precision cosmology. The physical reason behind this argument is the deep gravitational potential of big clusters $\left(M_{200} \gtrsim 3 \times 10^{14} \mathrm{M}_{\odot}\right)$. This makes it impossible for AGNs to expel gas today, in particular as the bubble enthalpy is poorly coupled to the thermal energy of the surrounding ICM which results in little heating and entropy generation of the central ICM on a buoyancy time scale (Pfrommer et al. 2012). Moreover, gas that was pushed beyond the virial radius by AGN feedback early-on gets pulled in during assembly of these big systems.

Similarly, we find almost no evolution of $f_{\text {star }}$ back to $z=1$ in our AGN feedback model. This implies that the stellar mass increases at the same rate as the DM mass, which is realized if most of the stellar mass is already in place by the time it assembles in the cluster halo (since we do not account for stellar mass loss in our simulations). This appears to agree with observations of the stellar mass assembly in clusters, although there is considerable uncertainty about the faint-end slope of the red sequence of cluster galaxies.

Cluster mergers may transfer energy from the DM component to the gas, potentially reducing $f_{\text {gas }}$ at the virial radius from its cosmic value. While our entropy-conserving SPH simulations suggest a value of $f_{\text {gas }} \simeq 0.93$ when scaled with the cosmic value, AMR simulations find somewhat larger values of $f_{\text {gas }} \simeq 0.97$ (Kravtsov et al. 2005). Radiative physics furthermore reduces $f_{\text {gas }}$ by locking baryons up into stars, causing a steeper slope of $f_{\text {gas }}$ towards the center. AGN feedback (or its quasar-like appearance) is most effective early-on during the assembly time of groups at $z \gtrsim 2$ and expels gas far beyond $R_{200}$ (McCarthy et al. 2011). This causes a much steeper dependence of $f_{\text {gas }}$ on cluster mass. At the same time, AGN feedback (in the incarnation that we modeled) is able to arrest overcooling and to drop the stellar mass by a factor of two As a result, our AGN feedback model keeps $f_{\text {star }}$ almost constant as a function of redshift (for $z \lesssim 1$ ) and mass, in particular for larger systems. This together implies a significantly increasing baryon fraction, $f_{\mathrm{b}}$, with cluster mass from 0.85 (at $M_{200} \simeq 10^{14} \mathrm{M}_{\odot}$ ) to 0.94 (at $M_{200} \simeq 10^{15} \mathrm{M}_{\odot}$ ). Those scalings of $f_{\text {gas }}$ and $f_{\mathrm{b}}$ need to be taken into account (e.g., through a marginalization process) when using X-ray and SZ surveys of cluster to constrain cosmological parameters. We emphasize that AGN feedback produces large effects on various thermodynamic quantities, including significant changes of stellar and gas mass fractions. While the qualitative results of our AGN feedback model are in line with approaches by other groups and produces robust predictions of the interesting parameter space preferred by observations, the detailed scalings and profiles may, however, depend on the particular model realization and could vary for different implementations.

We thank Volker Springel for enlightening discussions and careful reading the manuscript and thank Stefano Andreon, Dominique Eckert, Daisuke Nagai, Etienne Pointecouteau, Gabriel Pratt, and Greg Rudnick, Simon D.M. White for useful discussions. We thank our referee for providing an insightful report. C.P. gratefully acknowledges financial support of the Klaus Tschira Foundation. Research in Canada is supported by NSERC and CIFAR. Simulations were run on SCINET and CITA's Sunnyvale high-performance computing clusters. SCINET is funded and supported by CFI, NSERC, Ontario, ORF-RE and UofT deans. We also thank KITP for their hospitality during the 2011 galaxy cluster workshop. KITP is supported by National Science Foundation under Grant No. NSF PHY05-51164.

\section{REFERENCES}

Akamatsu, H., Hoshino, A., Ishisaki, Y., Ohashi, T., Sato, K., Takei, Y., \& Ota, N. 2011, PASJ, 63, 1019

Allen, S. W., Rapetti, D. A., Schmidt, R. W., Ebeling, H., Morris, R. G., \& Fabian, A. C. 2008, MNRAS, 383, 879

Allen, S. W., Schmidt, R. W., Ebeling, H., Fabian, A. C., \& van Speybroeck, L. 2004, MNRAS, 353, 457

Allen, S. W., Schmidt, R. W., \& Fabian, A. C. 2002, MNRAS, 334, L11

Andreon, S. 2006, A\&A, 448, 447

-. 2008, MNRAS, 386, 1045

Arnaud, M., Pointecouteau, E., \& Pratt, G. W. 2007, A\&A, 474, L37

Arnaud, M., Pratt, G. W., Piffaretti, R., Böhringer, H., Croston, J. H., \& Pointecouteau, E. 2010, A\&A, 517, A92

Battaglia, N., Bond, J. R., Pfrommer, C., \& Sievers, J. L. 2012a, ApJ, 758, 74

-. 2012b, ApJ, 758, 75

-. 2013a, in prep.

-. 2013b, in prep.

Battaglia, N., Bond, J. R., Pfrommer, C., Sievers, J. L., \& Sijacki, D. 2010, ApJ, 725, 91

Battye, R. A., \& Weller, J. 2003, Phys. Rev. D, 68, 083506

Bautz, M. W. et al. 2009, PASJ, 61, 1117

\footnotetext{
5 This suppression factor would be larger if we had run at higher resolution since models without AGN feedback do not produce numerically converged results (at the resolution that we could afford running our cosmological boxes).
} 
Bialek, J. J., Evrard, A. E., \& Mohr, J. J. 2001, ApJ, 555, 597

Booth, C. M., \& Schaye, J. 2009, MNRAS, 398, 53

Borgani, S., \& Kravtsov, A. 2009, arXiv:0906.4370

Bower, R. G., Benson, A. J., \& Crain, R. A. 2012, MNRAS, 422, 2816

Broderick, A. E., Chang, P., \& Pfrommer, C. 2012, ApJ, 752, 22

Brodwin, M. et al. 2006, ApJ, 651, 791

Carlstrom, J. E. et al. 2011, PASP, 123, 568

Chang, P., Broderick, A. E., \& Pfrommer, C. 2012, ApJ, 752, 23

Churazov, E. et al. 2012, MNRAS, 421, 1123

De Lucia, G. et al. 2004, ApJ, 610, L77

-. 2007, MNRAS, 374, 809

De Propris, R., Phillipps, S., \& Bremer, M. 2013, arXiv:1307.1592

Dubois, Y., Devriendt, J., Slyz, A., \& Teyssier, R. 2010, MNRAS, 409, 985

Duffy, A. R., Schaye, J., Kay, S. T., \& Dalla Vecchia, C. 2008, MNRAS, 390, L64

Eckert, D., Ettori, S., Molendi, S., Vazza, F., \& Paltani, S. 2013, A\&A, 551, A23

Eckert, D. et al. 2012, A\&A, 541, A57

Eke, V. R., Navarro, J. F., \& Frenk, C. S. 1998, ApJ, 503, 569

Enßlin, T. A., Pfrommer, C., Springel, V., \& Jubelgas, M. 2007, A\&A, 473, 41

Ettori, S., Dolag, K., Borgani, S., \& Murante, G. 2006, MNRAS, 365, 1021

Ettori, S., Morandi, A., Tozzi, P., Balestra, I., Borgani, S., Rosati, P., Lovisari,

L., \& Terenziani, F. 2009, A\&A, 501, 61

Evrard, A. E. 1990, ApJ, 363, 349

Fabjan, D., Borgani, S., Tornatore, L., Saro, A., Murante, G., \& Dolag, K. 2010, MNRAS, 401, 1670

Fowler, J. W. et al. 2007, Appl. Opt., 46, 3444

Frenk, C. S. et al. 1999, ApJ, 525, 554

Galli, S., Bartlett, J. G., \& Melchiorri, A. 2012, Phys. Rev. D, 86, 043516

Gao, L., Navarro, J. F., Frenk, C. S., Jenkins, A., Springel, V., \& White, S. D. M. 2012, MNRAS, 425, 2169

George, M. R., Fabian, A. C., Sanders, J. S., Young, A. J., \& Russell, H. R. 2009, MNRAS, 395, 657

Gilbank, D. G., Yee, H. K. C., Ellingson, E., Gladders, M. D., Loh, Y.-S., Barrientos, L. F., \& Barkhouse, W. A. 2008, ApJ, 673, 742

Giodini, S. et al. 2009, ApJ, 703, 982

Gladders, M. D., \& Yee, H. K. C. 2000, AJ, 120, 2148

Gonzalez, A. H., Zabludoff, A. I., \& Zaritsky, D. 2005, ApJ, 618, 195

Gonzalez, A. H., Zaritsky, D., \& Zabludoff, A. I. 2007, ApJ, 666, 147

Górski, K. M., Hivon, E., Banday, A. J., Wandelt, B. D., Hansen, F. K., Reinecke, M., \& Bartelmann, M. 2005, ApJ, 622, 759

Guo, F., \& Oh, S. P. 2008, MNRAS, 384, 251

Hayashi, E., \& White, S. D. M. 2008, MNRAS, 388, 2

Hoshino, A. et al. 2010, PASJ, 62, 371

Hu, W. 2003, Phys. Rev. D, 67, 081304

Humphrey, P. J., Buote, D. A., Brighenti, F., Flohic, H. M. L. G., Gastaldello,

F., \& Mathews, W. G. 2012, ApJ, 748, 11

Jubelgas, M., Springel, V., Enßlin, T., \& Pfrommer, C. 2008, A\&A, 481, 33

Kawaharada, M. et al. 2010, ApJ, 714, 423

Kay, S. T., Thomas, P. A., Jenkins, A., \& Pearce, F. R. 2004, MNRAS, 355, 1091

Khedekar, S., Majumdar, S., \& Das, S. 2010, Phys. Rev. D, 82, 041301

Kravtsov, A. V., Nagai, D., \& Vikhlinin, A. A. 2005, ApJ, 625, 588

Kravtsov, A. V., Vikhlinin, A., \& Nagai, D. 2006, ApJ, 650, 128

LaRoque, S. J., Bonamente, M., Carlstrom, J. E., Joy, M. K., Nagai, D., Reese, E. D., \& Dawson, K. S. 2006, ApJ, 652, 917

Lau, E. T., Kravtsov, A. V., \& Nagai, D. 2009, ApJ, 705, 1129

Leauthaud, A., Tinker, J., Behroozi, P. S., Busha, M. T., \& Wechsler, R. H. 2011, ApJ, 738, 45

Leitner, S. N., \& Kravtsov, A. V. 2011, ApJ, 734, 48

Lin, Y.-T., Mohr, J. J., \& Stanford, S. A. 2003, ApJ, 591, 749

Loewenstein, M., Zweibel, E. G., \& Begelman, M. C. 1991, ApJ, 377, 392

Majumdar, S., \& Mohr, J. J. 2003, ApJ, 585, 603

-. 2004, ApJ, 613, 41

Mancone, C. L. et al. 2012, ApJ, 761, 141

Mantz, A., Allen, S. W., Ebeling, H., Rapetti, D., \& Drlica-Wagner, A. 2010a, MNRAS, 406, 1773

Mantz, A., Allen, S. W., \& Rapetti, D. 2010b, MNRAS, 406, 1805

Mantz, A., Allen, S. W., Rapetti, D., \& Ebeling, H. 2010c, MNRAS, 406, 1759

Martig, M., \& Bournaud, F. 2010, ApJ, 714, L275

McCarthy, I. G. et al. 2007, MNRAS, 376, 497

McCarthy, I. G., Schaye, J., Bower, R. G., Ponman, T. J., Booth, C. M., Vecchia, C. D., \& Springel, V. 2011, MNRAS, 412, 1965

McCarthy, I. G. et al. 2010, MNRAS, 406, 822
Miller, E. D., Bautz, M., George, J., Mushotzky, R., Davis, D., \& Henry, J. P. 2012, in American Institute of Physics Conference Series, Vol. 1427, American Institute of Physics Conference Series, ed. R. Petre, K. Mitsuda, \& L. Angelini, 13-20

Mohr, J. J., Fabricant, D. G., \& Geller, M. J. 1993, ApJ, 413, 492

Muanwong, O., Thomas, P. A., Kay, S. T., \& Pearce, F. R. 2002, MNRAS, 336, 527

Muzzin, A. et al. 2009, ApJ, 698, 1934

Nagai, D., \& Lau, E. T. 2011, ApJ, 731, L10

Navarro, J. F., Frenk, C. S., \& White, S. D. M. 1997, ApJ, 490, 493

Nelson, K., Rudd, D. H., Shaw, L., \& Nagai, D. 2012, ApJ, 751, 121

Papovich, C. et al. 2010, ApJ, 716, 1503

Pfrommer, C. 2013, arXiv:1303.5443

Pfrommer, C., Chang, P., \& Broderick, A. E. 2012, ApJ, 752, 24

Pfrommer, C., Enßlin, T. A., Springel, V., Jubelgas, M., \& Dolag, K. 2007, MNRAS, 378, 385

Pfrommer, C., Springel, V., Enßlin, T. A., \& Jubelgas, M. 2006, MNRAS, 367, 113

Pinzke, A., \& Pfrommer, C. 2010, MNRAS, 409, 449

Planck Collaboration et al. 2013a, arXiv:1303.5076

-. 2013b, A\&A, 550, A131

-. 2013c, subm.

Planelles, S., Borgani, S., Dolag, K., Ettori, S., Fabjan, D., Murante, G., \& Tornatore, L. 2013, MNRAS, 431, 1487

Poole, G. B., Babul, A., McCarthy, I. G., Fardal, M. A., Bildfell, C. J., Quinn, T., \& Mahdavi, A. 2007, MNRAS, 380, 437

Pratt, G. W. et al. 2010, A\&A, 511, A85

Pratt, G. W., Croston, J. H., Arnaud, M., \& Böhringer, H. 2009, A\&A, 498, 361

Puchwein, E., Pfrommer, C., Springel, V., Broderick, A. E., \& Chang, P. 2012, MNRAS, 423, 149

Puchwein, E., Sijacki, D., \& Springel, V. 2008, ApJ, 687, L53

Puchwein, E., \& Springel, V. 2013, MNRAS, 428, 2966

Puchwein, E., Springel, V., Sijacki, D., \& Dolag, K. 2010, MNRAS, 406, 936

Rapetti, D., Allen, S. W., Mantz, A., \& Ebeling, H. 2009, MNRAS, 400, 699

-. 2010, MNRAS, 406, 1796

Rasia, E. et al. 2006, MNRAS, 369, 2013

-. 2012, New Journal of Physics, 14, 055018

Rasia, E., Tormen, G., \& Moscardini, L. 2004, MNRAS, 351, 237

Reichardt, C. L. et al. 2012, ApJ, 755, 70

Reiprich, T. H. et al. 2009, A\&A, 501, 899

Roncarelli, M., Ettori, S., Borgani, S., Dolag, K., Fabjan, D., \& Moscardini, L. 2013, MNRAS, 432, 3030

Roncarelli, M., Ettori, S., Dolag, K., Moscardini, L., Borgani, S., \& Murante, G. 2006, MNRAS, 373, 1339

Rudnick, G. et al. 2009, ApJ, 700, 1559

Rudnick, G. H., Tran, K.-V., Papovich, C., Momcheva, I., \& Willmer, C. 2012, ApJ, 755, 14

Sadat, R. et al. 2005, A\&A, 437, 31

Sanders, J. S., \& Fabian, A. C. 2012, MNRAS, 421, 726

Sanderson, A. J. R., Ponman, T. J., Finoguenov, A., Lloyd-Davies, E. J., \& Markevitch, M. 2003, MNRAS, 340, 989

Sato, T. et al. 2012, PASJ, 64, 95

Sembolini, F., Yepes, G., De Petris, M., Gottlöber, S., Lamagna, L., \& Comis, B. 2013, MNRAS, 429, 323

Shaw, L. D., Nagai, D., Bhattacharya, S., \& Lau, E. T. 2010, ApJ, 725, 1452

Sievers, J. L. et al. 2013, arXiv:1301.0824

Sijacki, D., Pfrommer, C., Springel, V., \& Enßlin, T. A. 2008, MNRAS, 387, 1403

Sijacki, D., \& Springel, V. 2006, MNRAS, 366, 397

Sijacki, D., Springel, V., Di Matteo, T., \& Hernquist, L. 2007, MNRAS, 380, 877

Simionescu, A. et al. 2011, Science, 331, 1576

Springel, V. 2005, MNRAS, 364, 1105

Springel, V., \& Hernquist, L. 2003, MNRAS, 339, 289

Stanford, S. A., Eisenhardt, P. R., \& Dickinson, M. 1998, ApJ, 492, 461

Stott, J. P., Smail, I., Edge, A. C., Ebeling, H., Smith, G. P., Kneib, J.-P., \& Pimbblet, K. A. 2007, ApJ, 661, 95

Sun, M., Voit, G. M., Donahue, M., Jones, C., Forman, W., \& Vikhlinin, A. 2009, ApJ, 693, 1142

Tanaka, M., Goto, T., Okamura, S., Shimasaku, K., \& Brinkmann, J. 2004, AJ, 128,2677

Teyssier, R., Moore, B., Martizzi, D., Dubois, Y., \& Mayer, L. 2011, MNRAS, 618

Thompson, T. A., Quataert, E., \& Murray, N. 2005, ApJ, 630, 167

Trac, H., Bode, P., \& Ostriker, J. P. 2011, ApJ, 727, 94

Vazza, F., Brüggen, M., Gheller, C., \& Brunetti, G. 2012, MNRAS, 421, 3375 
Vazza, F., Brunetti, G., Gheller, C., Brunino, R., \& Brüggen, M. 2011a, A\&A, 529, A17

Vazza, F., Roncarelli, M., Ettori, S., \& Dolag, K. 2011b, MNRAS, 413, 2305 Vikhlinin, A. et al. 2009a, ApJ, 692, 1033

Vikhlinin, A., Kravtsov, A., Forman, W., Jones, C., Markevitch, M., Murray, S. S., \& Van Speybroeck, L. 2006, ApJ, 640, 691

Vikhlinin, A. et al. 2009b, ApJ, 692, 1060

Voit, G. M. 2005, Reviews of Modern Physics, 77, 207

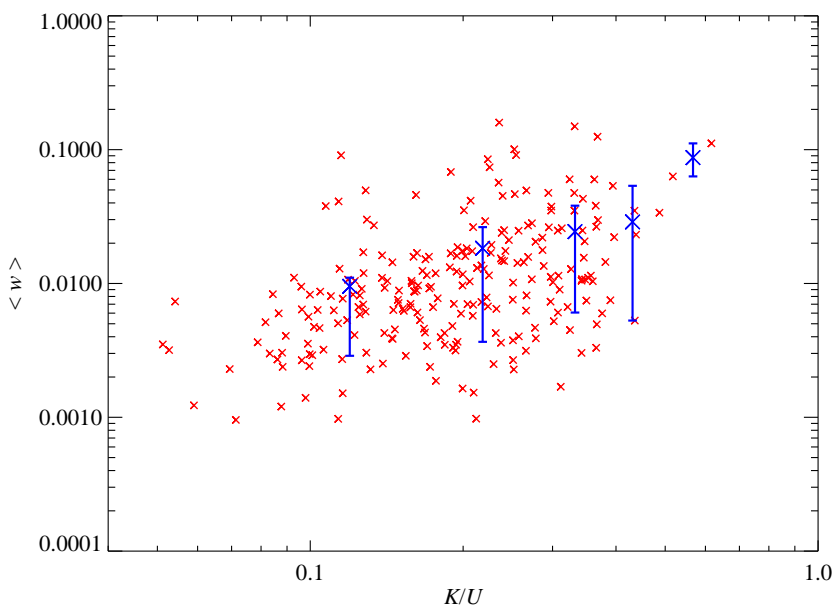

Figure 15. We compare the (theoretically accessible) kinetic-to-thermal energy ratio $K / U$ to the (observationally measurable) centroid shift $\langle w\rangle$ for the AGN feedback simulations at $z=0$. Here the red crosses represent those values for each cluster with $M_{200} \geq 2 \times 10^{14} \mathrm{M}_{\odot}$ in the simulations and the blue crosses are bin-averaged quantities. The linear correlation coefficient between $K / U$ and $\langle w\rangle$ is $r \sim 0.4$ indicating a weak correlation between these two dynamical cluster measures.

\section{APPENDIX}

\section{A. COMPARISON BETWEEN $K / U$ AND CENTROID SHIFT}

The kinetic-to-thermal energy ratio of a cluster, $K / U$, is the most direct quantity to estimate the dynamical activity of a cluster and easily calculable in simulations. However, it is not directly observationally accessible. As a result, other proxies have been introduced to estimate a cluster's dynamical state, such as the centroid shift $\langle w\rangle$ (Mohr et al. 1993), which measures the standard deviation between the projected separation of the X-ray peak and the centroid of the X-ray emission that is calculated in concentric circular apertures. In previous simulation work, $\langle w\rangle$ was found to be the most sensitive estimator of dynamical activity (Poole et al.|2007), thus motivating a comparison of the $K / U$ estimator for the dynamical state of a cluster to $\langle w\rangle$.

On projected X-ray surface brightness maps of our simulations, we follow Pratt et al. (2009) and calculate

$$
\langle w\rangle=\left[\frac{1}{N-1} \sum_{i=1}^{N}\left(\Delta_{i}-\langle\Delta\rangle\right)^{2}\right]^{1 / 2} \times \frac{1}{R_{200}},
$$

where $\Delta_{i}$ is the projected distance between the surface brightness peak and the centroid of the $i$ th aperture. The centroids are calculated within radial annuli ranging from $0.1 R_{200}$ to $0.05 i R_{200}$ with $i=3,4, \ldots, 20$. The central region is traditionally avoided to minimize biases associated with enhanced emission from cool cores (although inclusion does not significantly change the results obtained).

Figure 15 shows the correlation between $K / U$ and $\langle w\rangle$. Their correlation coefficient is $r \simeq 0.4$, which indicates that $K / U$ and $\langle w\rangle$ are only weakly correlated, making $\langle w\rangle$ a biased proxy for
Vulcani, B. et al. 2011, MNRAS, 412, 246

Walker, S. A., Fabian, A. C., Sanders, J. S., George, M. R., \& Tawara, Y. 2012, MNRAS, 422, 3503

Young, O. E., Thomas, P. A., Short, C. J., \& Pearce, F. 2011, MNRAS, 413, 691

Zibetti, S., White, S. D. M., Schneider, D. P., \& Brinkmann, J. 2005, MNRAS, 358, 949

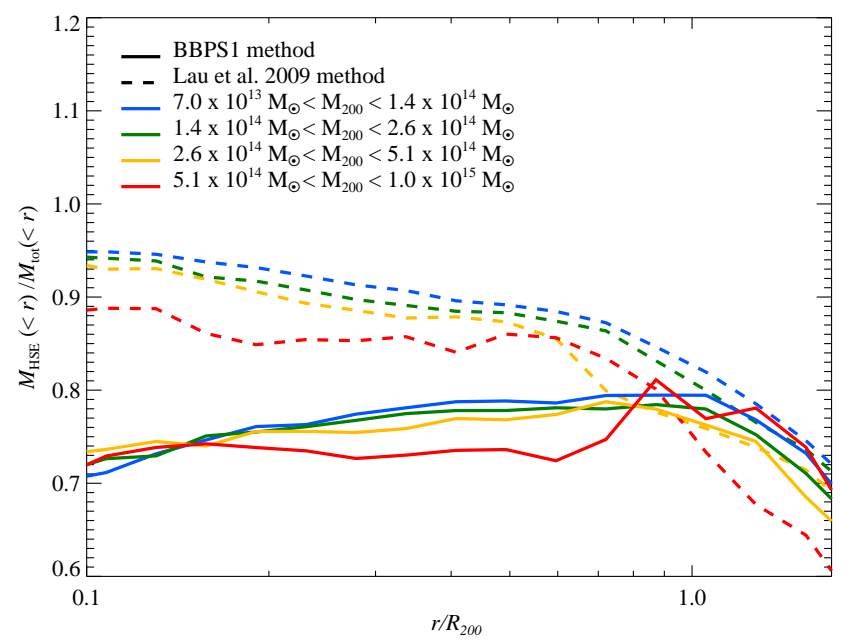

Figure 16. We show a comparison of two different methods for determining the HSE bias, $M_{\mathrm{HSE}} / M_{\mathrm{tot}}$, in various cluster mass bins for our AGN feedback simulations at $z=0$. The difference between $M_{\mathrm{HSE}} / M_{\mathrm{tot}}$ in this work (and BBPS1) and Lau et al. (2009) is the definition of $M_{\text {tot }}$. Here, we adopt $M_{\text {tot }} \equiv$ $M_{\text {DM }}+M_{\text {gas }}+M_{\text {star }}$ whereas Lau et al. (2009) define $\tilde{M}_{\text {tot }} \propto \mathrm{d}\left(P_{\mathrm{th}}+P_{\text {kin }}\right) / \mathrm{d} r$, which assumes spherical symmetry and a steady state. The radial trends differ the greatest at small radii, presumably due to violation of spherical symmetry and non-stationary, clumpy accretion.

the dynamical state of a cluster. At any given $K / U$, the values for $\langle w\rangle$ scatter by up to two orders of magnitude. This is in agreement with our previous work (BBPS1), where we showed that the correlation between $K U$ and $1-c / a$, the ratio of $3 \mathrm{D}$ major to minor axis, is $\simeq 0.58$. These two results suggest a reason for the weak correlation of the centroid shift with $K / U$. This is partially caused by the substantial scatter between the morphological irregularity and asphericity on the one side and dynamical activity of a cluster on the other side, and partially because of projection effects. More work is needed to combine different observationally accessible proxies that correlate more tightly with theoretical measures of dynamical cluster activity, such as the kinetic-to-thermal energy ratio, $K / U$.

\section{B. COMPARING DEFINITIONS FOR HYDROSTATIC MASS BIAS}

Here we compare various definitions for the bias by hydrostatic equilibrium assumption and review the differing methodologies and definitions used in the (simulation) literature. Direct comparison between our work and others is difficult since the methods used for calculating $M_{\mathrm{HSE}}$ are different. Previous simulation work used high resolution zoomed simulations of individual clusters (Rasia et al. 2006; Lau et al. 2009; Rasia et al. 2012), where the authors were able to smooth and removed clumps from each cluster before calculating $\mathrm{d} P_{\text {th }} / \mathrm{d} r$. Other simulations (e.g., Rasia et al. 2006) produced mock Xray images and performed similar smoothing and clump removal techniques to observations. These cleaning procedures were not preformed in this work (nor in BBPS1), because of the different goals of this work. We pursue a theoretically focused approach that should be equally applicable to X-ray (eROSITA) and SZ cluster surveys (by $A C T$ and $S P T$ ) and aim at strength- 
Table 1

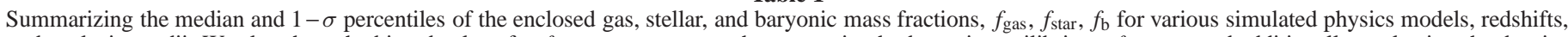

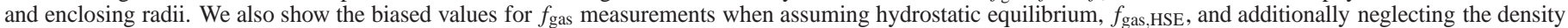
clumping, $f_{\text {gas,HSE+clump. }}$

\begin{tabular}{|c|c|c|c|c|c|c|c|c|c|}
\hline $1.4<M_{200} / 10^{14} \mathrm{M}_{\odot}<2.6$ & \multicolumn{3}{|c|}{$z=0$} & \multicolumn{3}{|c|}{$z=0.5$} & \multicolumn{3}{|c|}{$z=1$} \\
\hline Simulated physics $^{a}$ & $\mathrm{SH}$ & $\mathrm{CSF}$ & AGN & SH & $\mathrm{CSF}$ & AGN & SH & CSF & AGN \\
\hline \multicolumn{10}{|l|}{$r<R_{200}$} \\
\hline $\begin{array}{l}f_{\text {gas }} \\
f_{\text {gas,HSE }} \\
f_{\text {gas,HSE+clump }} \\
f_{\mathrm{b}}^{b} \\
f_{\text {star }}^{b} \\
\end{array}$ & $\begin{array}{c}0.93_{-0.03}^{+0.03} \\
1.12_{-0.14}^{+0.19} \\
1.23_{-0.16}^{+0.14} \\
0.93_{-0.03}^{+0.03} \\
- \\
\end{array}$ & $\begin{array}{l}0.76_{-0.03}^{+0.03} \\
0.96_{-0.11}^{+0.11} \\
1.06_{-0.15}^{+0.28} \\
0.95_{-0.03}^{+0.03} \\
0.19_{-0.02}^{+0.02} \\
\end{array}$ & $\begin{array}{l}0.75_{-0.04}^{+0.04} \\
0.89_{-0.12}^{+0.12} \\
0.95_{-0.12}^{+0.17} \\
0.86_{-0.04}^{+0.05} \\
0.11_{-0.02}^{+0.02} \\
\end{array}$ & $\begin{array}{c}0.94_{-0.03}^{+0.03} \\
1.15_{-0.21}^{+0.15} \\
1.30_{-0.32}^{+0.22} \\
0.94_{-0.03}^{+0.03} \\
- \\
\end{array}$ & $\begin{array}{l}0.79_{-0.02}^{+0.03} \\
1.04_{-0.18}^{+0.15} \\
1.17_{-0.19}^{+0.29} \\
0.96_{-0.03}^{+0.03} \\
0.16_{-0.02}^{+0.02} \\
\end{array}$ & $\begin{array}{l}0.75_{-0.05}^{+0.04} \\
0.90_{-0.15}^{+0.12} \\
1.01_{-0.16}^{+0.16} \\
0.86_{-0.05}^{+0.05} \\
0.11_{-0.02}^{+0.02} \\
\end{array}$ & $\begin{array}{c}0.94_{-0.01}^{+0.03} \\
1.15_{-0.21}^{+0.20} \\
1.36_{-0.28}^{+0.57} \\
0.94_{-0.01}^{+0.03} \\
- \\
\end{array}$ & $\begin{array}{l}0.80_{-0.01}^{+0.02} \\
1.05_{-0.19}^{+0.17} \\
1.26_{-0.24}^{+0.44} \\
0.96_{-0.02}^{+0.02} \\
0.16_{-0.01}^{+0.02} \\
\end{array}$ & $\begin{array}{l}0.73_{-0.03}^{+0.04} \\
0.87_{-0.10}^{+0.11} \\
1.00_{-0.14}^{+0.11} \\
0.83_{-0.04}^{+0.04} \\
0.10_{-0.02}^{+0.02} \\
\end{array}$ \\
\hline \multicolumn{10}{|l|}{$r<R_{500}$} \\
\hline $\begin{array}{l}f_{\text {gas }} \\
f_{\text {gas,HSE }} \\
f_{\text {gas,HSE+clump }} \\
f_{\mathrm{b}}^{b} \\
f_{\text {star }}^{b}\end{array}$ & $\begin{array}{c}0.92_{-0.04}^{+0.04} \\
1.12_{-0.11}^{+0.20} \\
1.21_{-0.14}^{+0.32} \\
0.92_{-0.04}^{+0.04} \\
-\end{array}$ & $\begin{array}{l}0.71_{-0.03}^{+0.03} \\
0.90_{-0.09}^{+0.15} \\
0.97_{-0.12}^{+0.24} \\
0.96_{-0.04}^{+0.04} \\
0.25_{-0.03}^{+0.03}\end{array}$ & $\begin{array}{l}0.69_{-0.05}^{+0.04} \\
0.82_{-0.09}^{+0.12} \\
0.87_{-0.10}^{+0.14} \\
0.83_{-0.05}^{+0.05} \\
0.14_{-0.03}^{+0.03}\end{array}$ & $\begin{array}{c}0.95_{-0.03}^{+0.03} \\
1.16_{-0.15}^{+0.18} \\
1.31_{-0.20}^{+0.26} \\
0.95_{-0.03}^{+0.03} \\
-\end{array}$ & $\begin{array}{l}0.75_{-0.02}^{+0.03} \\
0.97_{-0.11}^{+0.13} \\
1.09_{-0.16}^{+0.23} \\
0.97_{-0.03}^{+0.04} \\
0.22_{-0.02}^{+0.02}\end{array}$ & $\begin{array}{l}0.69_{-0.04}^{+0.05} \\
0.83_{-0.10}^{+0.14} \\
0.93_{-0.13}^{+0.15} \\
0.83_{-0.05}^{+0.06} \\
0.14_{-0.02}^{+0.03}\end{array}$ & $\begin{array}{c}0.95_{-0.04}^{+0.03} \\
1.12_{-0.15}^{+0.23} \\
1.28_{-0.22}^{+0.35} \\
0.95_{-0.04}^{+0.03} \\
-\end{array}$ & $\begin{array}{l}0.77_{-0.02}^{+0.02} \\
0.98_{-0.13}^{+0.18} \\
1.12_{-0.17}^{+0.28} \\
0.97_{-0.03}^{+0.04} \\
0.20_{-0.02}^{+0.03}\end{array}$ & $\begin{array}{l}0.68_{-0.04}^{+0.04} \\
0.79_{-0.10}^{+0.12} \\
0.89_{-0.12}^{+0.18} \\
0.80_{-0.05}^{+0.05} \\
0.13_{-0.02}^{+0.03}\end{array}$ \\
\hline \multicolumn{10}{|l|}{$r<R_{1000}$} \\
\hline $\begin{array}{l}f_{\text {gas }} \\
f_{\text {gas,HSE }} \\
f_{\text {gas,HSE+clump }} \\
f_{\mathrm{b}}^{b} \\
f_{\text {star }}^{b}\end{array}$ & $\begin{array}{c}0.90_{-0.05}^{+0.04} \\
1.10_{-0.09}^{+0.21} \\
1.16_{-0.10}^{+0.25} \\
0.90_{-0.05}^{+0.04} \\
-\end{array}$ & $\begin{array}{l}0.64_{-0.05}^{+0.05} \\
0.81_{-0.07}^{+0.14} \\
0.84_{-0.08}^{+0.17} \\
0.99_{-0.06}^{+0.06} \\
0.35_{-0.04}^{+0.04}\end{array}$ & $\begin{array}{l}0.60_{-0.06}^{+0.05} \\
0.74_{-0.09}^{+0.10} \\
0.77_{-0.10}^{+0.13} \\
0.79_{-0.06}^{+0.06} \\
0.19_{-0.05}^{+0.04}\end{array}$ & $\begin{array}{c}0.93_{-0.05}^{+0.05} \\
1.19_{-0.13}^{+0.22} \\
1.30_{-0.33}^{+0.19} \\
0.93_{-0.05}^{+0.05} \\
-\end{array}$ & $\begin{array}{l}0.69_{-0.05}^{+0.05} \\
0.91_{-0.12}^{+0.18} \\
0.99_{-0.14}^{+0.26} \\
1.00_{-0.06}^{+0.06} \\
0.30_{-0.04}^{+0.04}\end{array}$ & $\begin{array}{l}0.61_{-0.06}^{+0.06} \\
0.78_{-0.10}^{+0.10} \\
0.84_{-0.12}^{+0.17} \\
0.80_{-0.08}^{+0.08} \\
0.19_{-0.04}^{+0.05}\end{array}$ & $\begin{array}{c}0.95_{-0.04}^{+0.03} \\
1.24_{-0.22}^{+0.20} \\
1.31_{-0.35}^{+0.18} \\
0.95_{-0.04}^{+0.03} \\
-\end{array}$ & $\begin{array}{l}0.71_{-0.03}^{+0.04} \\
0.97_{-0.18}^{+0.18} \\
1.04_{-0.25}^{+0.20} \\
0.99_{-0.05}^{+0.07} \\
0.28_{-0.04}^{+0.06}\end{array}$ & $\begin{array}{l}0.61_{-0.05}^{+0.04} \\
0.75_{-0.09}^{+0.24} \\
0.82_{-0.10}^{+0.25} \\
0.79_{-0.07}^{+0.07} \\
0.17_{-0.05}^{+0.05}\end{array}$ \\
\hline
\end{tabular}

${ }^{a} \mathrm{SH} \equiv$ Shock heating, $\mathrm{CSF} \equiv$ Radiative cooling, AGN $\equiv$ AGN feedback

${ }^{b}$ Note that $f_{\text {star }}$ and $f_{\mathrm{b}}$ are similarly affected from the HSE bias as $f_{\text {gas }}$, which may increase the total baryon budget in galaxy clusters beyond the cosmic value.

Table 2

Summarizing the median and $1-\sigma$ percentiles of the enclosed gas, stellar, and baryonic mass fractions, $f_{\text {gas }}, f_{\text {star }}, f_{\mathrm{b}}$ for various cluster masses, redshifts, and enclosing radii in our AGN feedback model. We also show the biased values for $f_{\text {gas }}$ measurements when assuming hydrostatic equilibrium, $f_{\text {gas,HSE}}$, and additionally neglecting the density clumping, $f_{\text {gas, HSE+clump. }}$.

\begin{tabular}{|c|c|c|c|c|c|c|c|c|c|}
\hline \multirow[t]{2}{*}{ AGN feedback } & \multicolumn{3}{|c|}{$0.7<M_{200} / 10^{14} \mathrm{M}_{\odot}<1.4$} & \multicolumn{3}{|c|}{$101.4<M_{200} / 10^{14} \mathrm{M}_{\odot}<2.6$} & \multicolumn{3}{|c|}{$2.6<M_{200} / 10^{14} \mathrm{M}_{\odot}<5.1$} \\
\hline & $z=0$ & $z=0.5$ & $z=1.0$ & $z=0$ & $z=0.5$ & $z=1.0$ & $z=0$ & $z=0.5$ & $z=1.0$ \\
\hline \multicolumn{10}{|l|}{$r<R_{200}$} \\
\hline $\begin{array}{l}f_{\text {gas }} \\
f_{\text {gas,HSE }} \\
f_{\text {gas,HSE+clump }} \\
f_{\mathrm{b}}^{a} \\
f_{\text {star }}^{a} \\
\end{array}$ & $\begin{array}{l}0.72_{-0.05}^{+0.05} \\
0.86_{-0.12}^{+0.13} \\
0.91_{-0.13}^{+0.17} \\
0.79_{-0.06}^{+0.05} \\
0.11_{-0.03}^{+0.02} \\
\end{array}$ & $\begin{array}{l}0.72_{-0.05}^{+0.05} \\
0.88_{-0.15}^{+0.13} \\
0.95_{-0.14}^{+0.21} \\
0.80_{-0.06}^{+0.06} \\
0.11_{-0.02}^{+0.03} \\
\end{array}$ & $\begin{array}{l}0.69_{-0.06}^{+0.05} \\
0.86_{-0.16}^{+0.14} \\
0.96_{-0.18}^{+0.24} \\
0.78_{-0.07}^{+0.06} \\
0.11_{-0.03}^{+0.02} \\
\end{array}$ & $\begin{array}{l}0.75_{-0.04}^{+0.04} \\
0.89_{-0.12}^{+0.12} \\
0.95_{-0.12}^{+0.17} \\
0.83_{-0.04}^{+0.05} \\
0.11_{-0.02}^{+0.02} \\
\end{array}$ & $\begin{array}{l}0.75_{-0.05}^{+0.04} \\
0.90_{-0.15}^{+0.12} \\
1.01_{-0.17}^{+0.16} \\
0.83_{-0.05}^{+0.05} \\
0.11_{-0.02}^{+0.02} \\
\end{array}$ & $\begin{array}{l}0.73_{-0.03}^{+0.04} \\
0.87_{-0.10}^{+0.11} \\
1.00_{-0.14}^{+0.17} \\
0.80_{-0.04}^{+0.04} \\
0.10_{-0.02}^{+0.02} \\
\end{array}$ & $\begin{array}{l}0.77_{-0.03}^{+0.02} \\
0.92_{-0.09}^{+0.11} \\
1.02_{-0.13}^{+0.19} \\
0.86_{-0.03}^{+0.03} \\
0.11_{-0.02}^{+0.01} \\
\end{array}$ & $\begin{array}{l}0.78_{-0.03}^{+0.02} \\
0.92_{-0.11}^{+0.15} \\
1.04_{-0.14}^{+0.23} \\
0.86_{-0.04}^{+0.03} \\
0.10_{-0.01}^{+0.02} \\
\end{array}$ & $\begin{array}{l}0.78_{-0.03}^{+0.01} \\
0.90_{-0.12}^{+0.09} \\
1.00_{-0.03}^{+0.22} \\
0.90_{-0.03}^{+0.01} \\
0.11_{-0.01}^{+0.01} \\
\end{array}$ \\
\hline \multicolumn{10}{|l|}{$r<R_{500}$} \\
\hline $\begin{array}{l}f_{\text {gas }} \\
f_{\text {gas,HSE }} \\
f_{\text {gas,HSE+clump }} \\
f_{\mathrm{b}}^{a} \\
f_{\text {star }}^{a} \\
\end{array}$ & $\begin{array}{l}0.64_{-0.05}^{+0.06} \\
0.78_{-0.09}^{+0.11} \\
0.82_{-0.10}^{+0.14} \\
0.79_{-0.07}^{+0.07} \\
0.15_{-0.04}^{+0.03} \\
\end{array}$ & $\begin{array}{l}0.65_{-0.06}^{+0.05} \\
0.81_{-0.12}^{+0.13} \\
0.86_{-0.13}^{+0.19} \\
0.80_{-0.07}^{+0.07} \\
0.14_{-0.03}^{+0.05} \\
\end{array}$ & $\begin{array}{l}0.63_{-0.07}^{+0.07} \\
0.79_{-0.16}^{+0.13} \\
0.86_{-0.14}^{+0.17} \\
0.78_{-0.08}^{+0.07} \\
0.14_{-0.03}^{+0.03} \\
\end{array}$ & $\begin{array}{l}0.69_{-0.05}^{+0.04} \\
0.82_{-0.09}^{+0.12} \\
0.87_{-0.10}^{+0.14} \\
0.83_{-0.06}^{+0.05} \\
0.14_{-0.03}^{+0.03} \\
\end{array}$ & $\begin{array}{l}0.69_{-0.04}^{+0.05} \\
0.83_{-0.10}^{+0.14} \\
0.93_{-0.13}^{+0.15} \\
0.83_{-0.05}^{+0.06} \\
0.14_{-0.02}^{+0.03} \\
\end{array}$ & $\begin{array}{l}0.68_{-0.04}^{+0.04} \\
0.79_{-0.10}^{+0.12} \\
0.89_{-0.12}^{+0.18} \\
0.80_{-0.05}^{+0.05} \\
0.13_{-0.02}^{+0.03} \\
\end{array}$ & $\begin{array}{l}0.73_{-0.04}^{+0.03} \\
0.88_{-0.11}^{+0.17} \\
0.92_{-0.09}^{+0.21} \\
0.86_{-0.04}^{+0.04} \\
0.13_{-0.02}^{+0.02} \\
\end{array}$ & $\begin{array}{l}0.73_{-0.04}^{+0.03} \\
0.87_{-0.14}^{+0.11} \\
0.98_{-0.17}^{+0.11} \\
0.86_{-0.04}^{+0.04} \\
0.12_{-0.02}^{+0.02} \\
\end{array}$ & $\begin{array}{l}0.76_{-0.02}^{+0.01} \\
0.89_{-0.09}^{+0.13} \\
1.07_{-0.23}^{+0.07} \\
0.90_{-0.03}^{+0.02} \\
0.14_{-0.01}^{+0.01} \\
\end{array}$ \\
\hline \multicolumn{10}{|l|}{$r<R_{1000}$} \\
\hline $\begin{array}{l}f_{\text {gas }} \\
f_{\text {gas,HSE }} \\
f_{\text {gas,HSE+clump }} \\
f_{\mathrm{b}}^{a} \\
f_{\text {star }}^{a}\end{array}$ & $\begin{array}{l}0.55_{-0.07}^{+0.06} \\
0.69_{-0.09}^{+0.10} \\
0.71_{-0.09}^{+0.11} \\
0.75_{-0.09}^{+0.08} \\
0.20_{-0.06}^{+0.05}\end{array}$ & $\begin{array}{l}0.56_{-0.07}^{+0.06} \\
0.72_{-0.11}^{+0.16} \\
0.76_{-0.12}^{+0.19} \\
0.76_{-0.09}^{+0.09} \\
0.20_{-0.06}^{+0.06}\end{array}$ & $\begin{array}{l}0.55_{-0.07}^{+0.07} \\
0.71_{-0.11}^{+0.17} \\
0.79_{-0.15}^{+0.20} \\
0.75_{-0.09}^{+0.08} \\
0.20_{-0.05}^{+0.05}\end{array}$ & $\begin{array}{c}0.60_{-0.06}^{+0.05} \\
0.74_{-0.09}^{+0.10} \\
0.77_{-0.10}^{+0.13} \\
0.79_{-0.07}^{+0.06} \\
0.19_{-0.05}^{+0.04}\end{array}$ & $\begin{array}{c}0.61_{-0.06}^{+0.06} \\
0.78_{-0.10}^{+0.10} \\
0.84_{-0.12}^{+0.17} \\
0.80_{-0.08}^{+0.08} \\
0.19_{-0.04}^{+0.05}\end{array}$ & $\begin{array}{c}0.61_{-0.05}^{+0.04} \\
0.75_{-0.09}^{+0.24} \\
0.82_{-0.10}^{+0.25} \\
0.79_{-0.07}^{+0.07} \\
0.17_{-0.05}^{+0.05}\end{array}$ & $\begin{array}{c}0.66_{-0.05}^{+0.05} \\
0.80_{-0.08}^{+0.11} \\
0.84_{-0.08}^{+0.14} \\
0.84_{-0.06}^{+0.06} \\
0.18_{-0.04}^{+0.03}\end{array}$ & $\begin{array}{c}0.67_{-0.06}^{+0.03} \\
0.84_{-0.10}^{+0.11} \\
0.89_{-0.09}^{+0.15} \\
0.83_{-0.06}^{+0.06} \\
0.16_{-0.02}^{+0.05}\end{array}$ & $\begin{array}{c}0.67_{-0.03}^{+0.03} \\
0.97_{-0.10}^{+0.11} \\
1.06_{-0.15}^{+0.11} \\
0.86_{-0.03}^{+0.04} \\
0.19_{-0.01}^{+0.03}\end{array}$ \\
\hline
\end{tabular}

${ }^{a}$ Note that $f_{\text {star }}$ and $f_{\mathrm{b}}$ are similarly affected from the HSE bias as $f_{\text {gas }}$, which may increase the total baryon budget in galaxy clusters beyond the cosmic value. 
ening the understanding of the underlying connection of the hot ICM rather than producing a specific mock observation tailored to a particular observatory.

Another difference is in the definition of $M_{\text {tot }}$. Lau et al. (2009) define the total mass as $\tilde{M}_{\text {tot }} \propto \mathrm{d}\left(P_{\text {th }}+P_{\text {kin }}\right) / \mathrm{d} r$, which assumes spherical symmetry and a steady state. This is different from our approach (and that in BBPS1) since we define $M_{\mathrm{tot}} \equiv M_{\mathrm{DM}}+M_{\mathrm{gas}}+M_{\mathrm{star}}$. These different definitions for $M_{\mathrm{tot}}$ produce significantly different HSE biases, $M_{\mathrm{HSE}} / M_{\mathrm{tot}}$, within $R_{200}$ as illustrated in Figure 16. Note that the difference of the solid and dashed curves in Figure 16 (at each color and radius) directly measures $\Delta M_{\text {tot }}=M_{\text {tot }}-\tilde{M}_{\text {tot }}$ (since we use the identical $M_{\mathrm{HSE}}$ in both cases). In particular both approaches yield significantly different radial trends at small radii, presumably due to violation of spherical symmetry and non-stationary, clumpy accretion in the definition of $\tilde{M}_{\text {tot }}$. Adopting the definition of Lau et al. (2009) for the total mass, we recover the same radial trends as these authors, which suggests that the main difference originates from the different definition of $M_{\text {tot }}$ rather than $M_{\text {HSE }}$.

\section{HORIZONTALLY SLICED SPH KERNEL INTEGRAL}

To speed up the mass binning of a distribution of SPH particles into a spherical shell, we integrate the mass portion of the SPH-kernel within that shell. We make the approximation that the smoothing length $h$ is much smaller than the radius of the shell in question, $r_{\text {shell }}$, so the shell can be thought of as two plane-parallel slices. We obtain a $9^{\text {th }}$ order polynomial fit to the integrated SPH mass fraction below some normalized distance $x$, which is constrained to be exact at $x=\{-1,0,1\}$ :

$$
\begin{aligned}
M_{\mathrm{gas}}(<x) & =0.5+1.391850 x-2.543398 x^{3} \\
& +3.373049 x^{5}-2.474134 x^{7}+0.7526237 x^{9}
\end{aligned}
$$

where $x=\left(r-r_{0}\right) / h$ for an SPH particle at position $x_{0}$. The fit is everywhere accurate to better than $2 \times 10^{-3}$ of the total mass. Clearly, this approximation breaks down near the center of the cluster (where the shell radius could become comparable to the smoothing length). However, the constraint at \pm 1 guarantees conservation of mass even in the event the approximation $r_{\text {shell }} \gg h$ breaks down.

\section{VARIANCE OF PROJECTED $M_{\mathrm{GAS}}$ AND $F_{\mathrm{GAS}}$ FOR DIFFERING NUMBER OF SECTOR ELEMENTS}

In Figure 17, we address how the 2D angular variances of $M_{\text {gas }}$ and $f_{\text {gas }}$ change if we vary the number of sector elements. There is almost no difference in $\sigma_{M_{\mathrm{gas}}}$ for varying number of sector elements and the small systematic decrease in $\sigma_{f_{\mathrm{gas}}}$ is expected for the increase in bin volume (decrease in sector numbers). Note that this is quite different for the angular variance of the X-ray luminosity, which strongly depends on the number of sector elements, especially in SPH simulations (see Figure 4 of Vazza et al. 2011b). We believe that this increased scatter solely derives from the increased clumping factor with radius (see Figure1). 
BATTAGLIA ET AL.
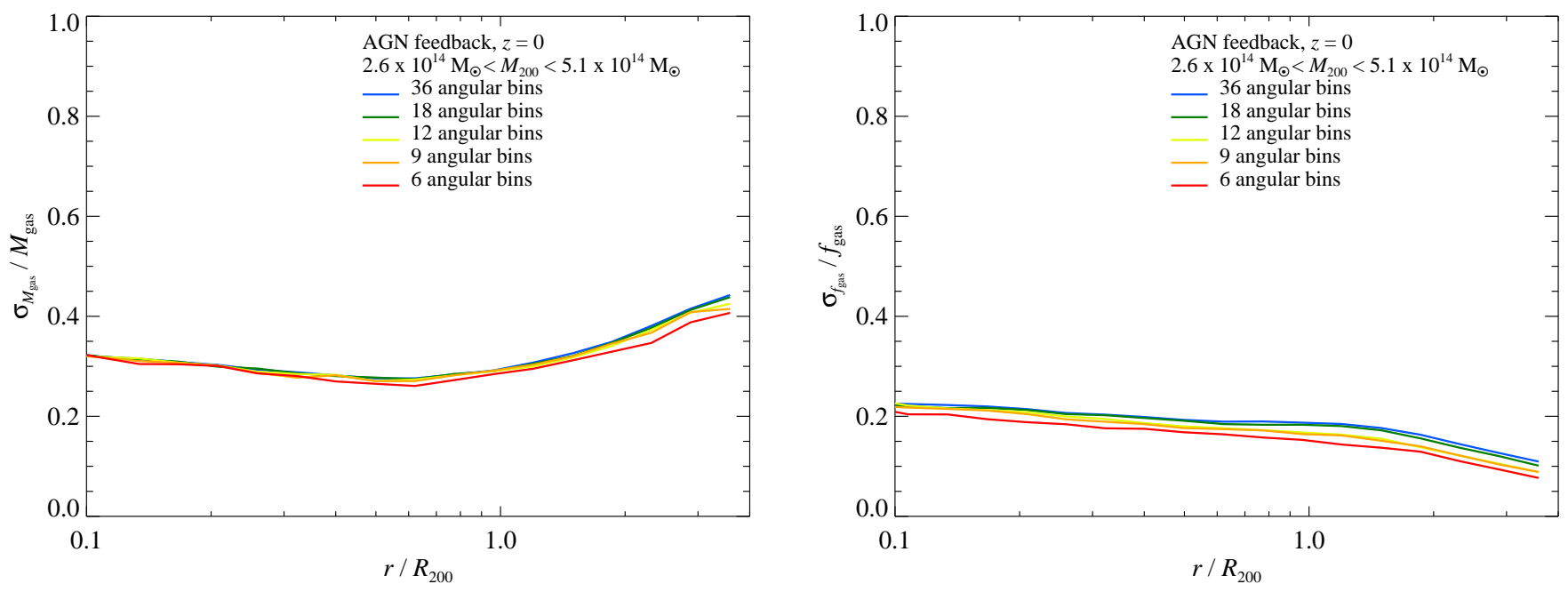

Figure 17. Angular variance in projected (2D) maps of $M_{\mathrm{gas}}$ (left) and $f_{\mathrm{gas}}$ (right) for differing number of sector elements. Here we show the measured variance (uncorrected for the sub-Poissonian noise term) and use all clusters in our sample with virial masses in the range $2.6 \times 10^{14} \mathrm{M}_{\odot}<M_{200}<5.1 \times 10^{14} \mathrm{M}_{\odot}$. There is only little difference in the $2 \mathrm{D}$ angular variances for varying number of sector elements. 